\title{
Asymmetric Organocatalytic Synthesis of Highly Functionalized Spirocyclohexane Indandiones via a One-Pot Michael/Michael/Aldol Sequence
}

\author{
Marcus Blümel ${ }^{a}$ \\ Pankaj Chauhan ${ }^{a}$ \\ Cornelia Vermeeren ${ }^{a}$ \\ Angelika Dreier ${ }^{b}$ \\ Christian Lehmann ${ }^{b}$ \\ Dieter Enders*a \\ a Institute of Organic Chemistry, RWTH Aachen University, \\ Landoltweg 1, 52074 Aachen, Germany \\ enders@rwth-aachen.de \\ b Chemical Crystallography and Electron Microscopy, Max- \\ Planck-Institut für Kohlenforschung, Kaiser-Wilhelm-Platz 1 , \\ 45470 Mülheim an der Ruhr, Germany \\ lehmann@mpi-muelheim.mpg.de
}
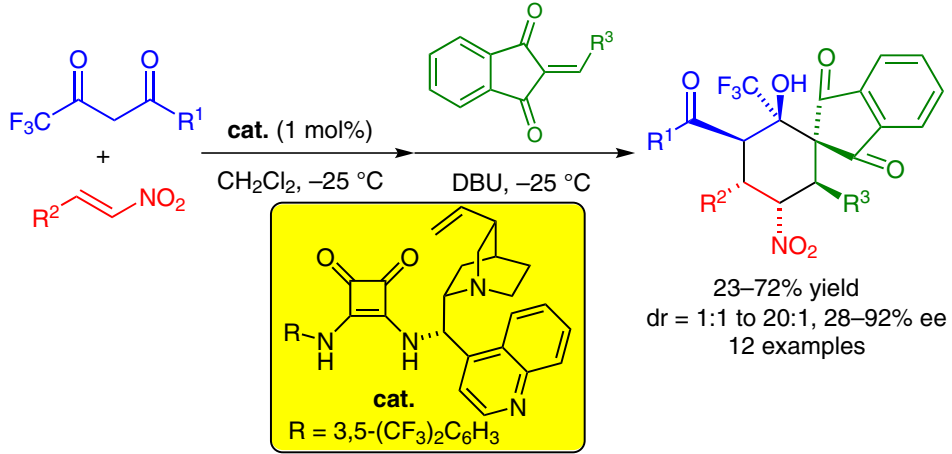

$23-72 \%$ yield

$\mathrm{dr}=1: 1$ to $20: 1,28-92 \%$ ee

12 examples
Received: 24.07.2015

Accepted: 27.07.2015

Published online: 14.08 .2015

DOI: 10.1055/s-0035-1560072; Art ID: ss-2015-z0457-op

License terms: CC $\Theta \$$

Abstract The asymmetric organocatalytic one-pot Michael/Michael/ aldol reaction of trifluoromethyl-substituted 1,3-dicarbonyl compounds, nitroolefins, and 2-arylidene indandiones catalyzed sequentially by a cinchona-derived squaramide and DBU leads to spirocyclohexaneindan-1,3-diones bearing five adjacent stereogenic centers including a trifluoromethylated one in medium to very good yields and enantioselectivities, but generally in low to high diastereomeric ratios.

Key words organocatalysis, one-pot reaction, squaramide, trifluoromethyl group, spirocycles

Organocatalytic one-pot transformations have emerged as a viable tool to generate important key structures of naturally occurring compounds and drug candidates, for example, five- and six-membered carbo- and heterocycles. ${ }^{1}$ An interesting class of carbocyclic compounds is that of the spiroindanone derivatives. Selected examples of natural products and potential drugs containing the spirocyclohexane-indanone core are depicted in Figure $10^{2,3}$ The coleophomone diterpenes I and II exhibit various interesting biological activities such as antibacterial and antifungal properties. ${ }^{3}$ Furthermore, they inactivate several proteases, for example, the heart chymase, and therefore a pharmaceutical application for the treatment of hypertension and congestive heart failure seems feasible. ${ }^{2 b, 3}$ The spirocyclic cyclohexanone indandione III is a potential lead for the development of new anticancer agents. ${ }^{4}$
In pharmaceutical research, not only the in vivo activity but also other properties like lipophilicity, bioavailability, binding selectivity, and metabolic stability of new drugs are highly important. Therefore, it is very common to test trifluoromethyl derivatives of promising drug candidates to improve the biological activities of these compounds. As outlined in recently published comprehensive reviews, there are many trifluoromethyl-substituted drugs on the market, but there is still a need to develop novel enantioselective catalytic reactions for the formation of key structures bearing the trifluoromethyl substituent. ${ }^{5}$<smiles>CC(C)=CCC1(CC=C(C)C)C(=O)[C@@]2(CCOc3cccc(O)c3C2=O)C(=O)C=C1C</smiles>

I coleophomone A<smiles>[2H]C1CC(=O)C[C@@H](c2ccccc2)C12C(=O)c1ccccc1C2=O</smiles>

III<smiles>COc1cccc2c1[C@H](O)C1(C(=O)C=C(C)C(CC=C(C)C)(CC=C(C)C)C1=O)C2=O</smiles>

II<smiles>[Y6]c1ccc(-c2ccc(CO)cc2)cc1</smiles>

Figure 1 Selected examples of natural products containing a spirocyclohexane indanone scaffold (red) ${ }^{2}$ 
There are several reports on the organocatalytic construction of spirocyclohexane-indan-1,3-diones, but only a few on the enantioselective formation of this molecular backbone. ${ }^{6}$ During our work He, Wang, Peng, and co-workers described the synthesis of cyclohexane-indan-1,3-diones from aldehydes, nitroolefins, and arylidene indandiones with a TMS-prolinol catalyst (Scheme 1, top). ${ }^{6 \mathrm{k}}$ In 2009 Zhu, Song, and co-workers reported on a base-catalyzed Michael/Michael/aldol domino reaction leading to a racemic mixture of trifluoromethyl-substituted cyclohexanes bearing two indan-1,3-dione moieties (Scheme 1, middle). ${ }^{7}$ Despite the occurrence of spiroindanones in bioactive compounds and the importance of enantioenriched trifluoromethylated compounds as various drugs, there is no report on the asymmetric synthesis of structures combining both moieties. We envisioned a new route to compounds combining the spirocyclohexane indan-1,3-dione core with a tetrasubstituted stereogenic center bearing the $\mathrm{CF}_{3}$ substituent by a cinchona-derived squaramide-catalyzed Michael/Michael/aldol one-pot reaction. In contrast to the work of Zhu, Song, and co-workers, ${ }^{7}$ the new protocol would allow not only for the introduction of a trifluoromethyl group, but also for a more diverse substitution pattern in an asymmetric version (Scheme 1, bottom).

Based on the previous results of Rawal and co-workers, ${ }^{8}$ other groups, ${ }^{9}$ as well as our own studies,,$^{10}$ the Michael addition of the $\beta$-keto ester 1a to trans- $\beta$-nitrostyrene (2a) was carried out in the presence of the bifunctional squaramide catalyst $\mathbf{A}$ to afford the Michael adduct 3a. Since the enantiomeric excess could not be determined for $\mathbf{3 a}$, the maximum enantiomeric excess of the resulting final cyclohexane 5a was searched. ${ }^{11}$ Then we started our investigation by optimizing the reaction conditions for the reaction of the Michael adduct 3a and the $\alpha, \beta$-unsaturated indandione 4a (Table 1). The Michael/aldol domino reaction in $\mathrm{CH}_{2} \mathrm{Cl}_{2}$ did not proceed in the absence of a base additive (Table 1 , entry 1 ). Consequently, different organic and inorganic bases were added to test the impact on the outcome of the reaction. To our delight, the reaction worked in the presence of an additional base to provide the cyclohexane $\mathbf{5 a}$ in moderate to very good yields (entries 2-9). In contrast to the inorganic bases $\mathrm{K}_{2} \mathrm{CO}_{3}$ and $\mathrm{KOAc}$ (entries 8, 9), all organic bases tested led to no significant loss of the enantiomeric excess, however, the yield varied strongly depending on the nature of the base. In the case of DABCO, TBD, and DIPEA, a third diastereomer was observed (entries 3, 5, and 6 ). Since DBU (entry 7) provided the best yield (71\%) and the best enantiomeric excess (89\%) it was used for the subsequent optimization by solvent screening testing various polar and nonpolar solvents. Whereas in the case of $\mathrm{CHCl}_{3}$, $\mathrm{CCl}_{4}$, and EtOAc the enantiomeric excess of $\mathbf{5 a}$ dropped to $85 \%, 80 \%$, and $85 \%$, respectively (entries, $10,11,16$ ), the yield decreased in the case of toluene, $\mathrm{Et}_{2} \mathrm{O}, \mathrm{THF}$, and $\mathrm{MeCN}$
He, Wang, Peng, and co-workers: ${ }^{6 k}$

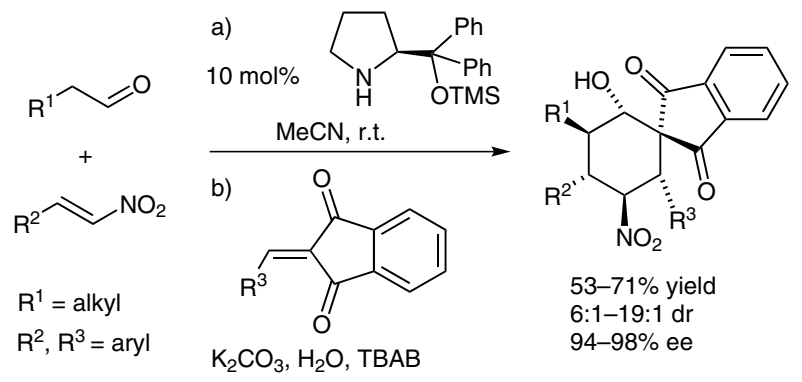

Zhu, Song, and co-workers: ${ }^{7}$

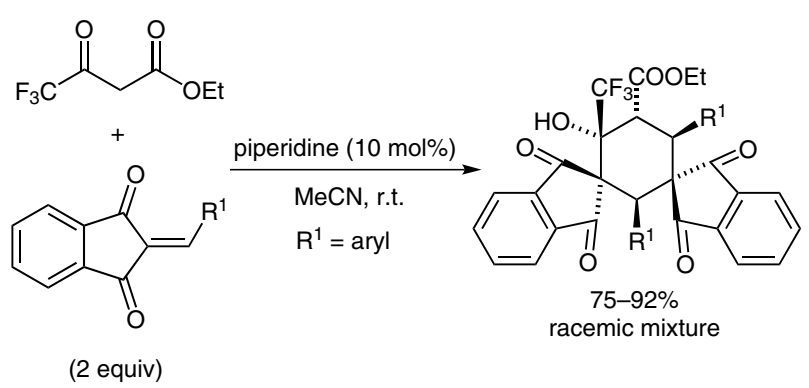

our work:

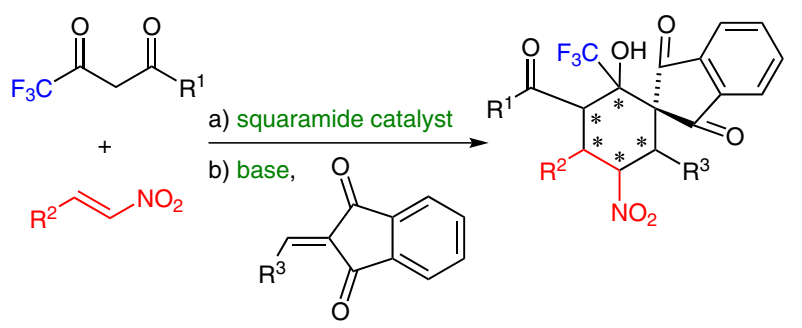

- formation of a tetrasubstituted carbon bearing a $\mathrm{CF}_{3}$ group
- readily available substrates (e.g. nitroolefins)
- asymmetric organocatalytic one-pot sequence

Scheme 1 One-pot Michael/Michael/aldol sequences to spirocyclohexane indandione scaffolds

as reaction medium (entries 12-15). As a consequence, $\mathrm{CH}_{2} \mathrm{Cl}_{2}$ was found to be the best solvent (entry 7), and we started to investigate the one-pot procedure starting from $\beta$-keto ester 1a and nitrostyrene $\mathbf{2 a}$ in $\mathrm{CH}_{2} \mathrm{Cl}_{2}$ in the presence of different catalysts using DBU as base in the second step (Table 2).

All tested cinchona-derived squaramides A-G as well as the thiourea catalyst $\mathbf{H}$ were capable of catalyzing the onepot reaction (Table 2, entries 1-8), albeit with very different results. The catalysts $\mathbf{A}-\mathbf{D}$ gave high enantioselectivities, whereas the enantioselectivities achieved by the quinidineand cinchonine-based squaramides $\mathbf{E}-\mathbf{G}$ as well as the thiourea $\mathbf{H}$ were merely moderate. In order to optimize the yield and the stereoselectivity, the catalyst loading of $\mathbf{B}$ and C was increased. Interestingly, the use of both catalysts at higher catalyst loading resulted in a drop in yield and 
Table 1 Optimization of the Reaction Conditions ${ }^{\mathrm{a}}$

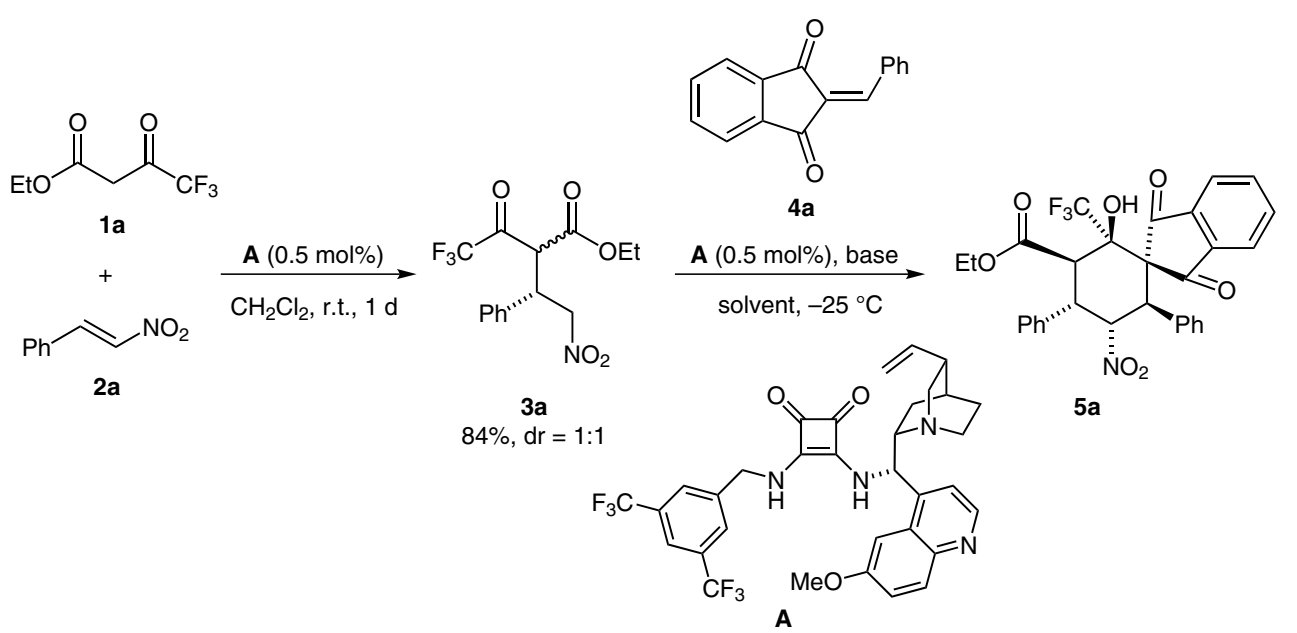

\begin{tabular}{|c|c|c|c|c|c|}
\hline Entry & Base & Solvent & Yield (\%) & $\mathrm{dr}^{\mathrm{c}}$ & ee $(\%)^{d}$ \\
\hline 1 & - & $\mathrm{CH}_{2} \mathrm{Cl}_{2}$ & NR & - & - \\
\hline 2 & $\mathrm{Et}_{3} \mathrm{~N}$ & $\mathrm{CH}_{2} \mathrm{Cl}_{2}$ & 56 & $1.2: 1$ & 89 \\
\hline 3 & DABCO & $\mathrm{CH}_{2} \mathrm{Cl}_{2}$ & $32^{\mathrm{e}}$ & $1.1: 1$ & 87 \\
\hline 4 & TMEDA & $\mathrm{CH}_{2} \mathrm{Cl}_{2}$ & 57 & $1.1: 1$ & 89 \\
\hline 5 & TBD & $\mathrm{CH}_{2} \mathrm{Cl}_{2}$ & $39^{e}$ & $1.1: 1$ & 88 \\
\hline 6 & DIPEA & $\mathrm{CH}_{2} \mathrm{Cl}_{2}$ & $44^{e}$ & 2.6:1 & 89 \\
\hline 7 & DBU & $\mathrm{CH}_{2} \mathrm{Cl}_{2}$ & 71 & 1:1.1 & 89 \\
\hline 8 & $\mathrm{~K}_{2} \mathrm{CO}_{3}$ & $\mathrm{CH}_{2} \mathrm{Cl}_{2}$ & 19 & $1: 3.9$ & 76 \\
\hline 9 & KOAC & $\mathrm{CH}_{2} \mathrm{Cl}_{2}$ & 22 & $1: 2.8$ & 28 \\
\hline 10 & DBU & $\mathrm{CHCl}_{3}$ & 50 & $1.2: 1$ & 85 \\
\hline 11 & DBU & $\mathrm{CCl}_{4}$ & 62 & $1: 1$ & 80 \\
\hline 12 & DBU & toluene & 63 & $1.7: 1$ & 90 \\
\hline 13 & DBU & $\mathrm{Et}_{2} \mathrm{O}$ & 36 & $1.4: 1$ & 90 \\
\hline 14 & DBU & THF & 49 & 2.1:1 & 90 \\
\hline 15 & DBU & $\mathrm{MeCN}$ & 65 & $1.4: 1$ & 91 \\
\hline 16 & DBU & EtOAc & 51 & $1.3: 1$ & 85 \\
\hline
\end{tabular}

${ }^{a}$ Reactions were carried out with $3 a(0.5 \mathrm{mmol}), \mathbf{A}(2.5 \mu \mathrm{mol}, 0.5 \mathrm{~mol} \%), 4 a(1.0 \mathrm{mmol}, 2$ equiv), and base $(0.1 \mathrm{mmol}, 0.2$ equiv) in solvent $(0.6 \mathrm{~mL}, c=0.8 \mathrm{M})$.

The enantiomeric excess of $\mathbf{3 a}$ could not be determined due to the critical instability under the applied HPLC conditions.

b Yield of the isolated product after column chromatography.

' Ratio of $(2 R, 3 R, 4 S, 5 R, 6 R)-5 \mathbf{a} /(2 S, 3 S, 4 S, 5 R, 6 R)$-5a determined by ${ }^{1} \mathrm{H}$ NMR spectroscopy.

${ }^{d}$ Enantiomeric excess of the major diastereomer determined by HPLC on a chiral stationary phase.

e The formation of one additional diastereomer was observed.

slightly increased ee values (entries 9, 10). As a consequence of the better catalytic activity of $\mathbf{C}$ at a catalyst loading of $1 \mathrm{~mol} \%$, $\mathbf{C}$ was used for the next optimizations (entry 14). A variation of the amount of the base or of the second Michael acceptor 4a did not have a beneficial effect on the results (entries 15-18). So, the optimized conditions include $1 \mathrm{~mol} \%$ of catalyst $\mathbf{C}$ in $\mathrm{CH}_{2} \mathrm{Cl}_{2}$ with 0.2 equivalent of DBU and 2.0 equivalents of alkene 4 at $-25^{\circ} \mathrm{C}$.
Having determined the optimal reaction conditions, the scope of the new one-pot reaction was extended by varying the substituents at the nitroalkene 2 . Electron-poor as well as electron-rich aromatic nitroalkenes underwent the reaction to give the corresponding spirocyclohexane indandiones $\mathbf{5}$ in good yields and high enantioselectivities (Table 3, 5b-h). Substituents in ortho-position to the nitrovinyl moiety and sterically demanding aromatic and heteroaromatic substituents resulted in an increased stereoselectivity of the one-pot reaction (Table 3, 5b, f, g, h). However, in the 
Table 2 Optimization of the One-Pot Michael/Michael/Aldol Reaction ${ }^{\mathrm{a}}$<smiles>CCOC(=O)CC(=O)C(F)(F)F</smiles>

2a

cat.

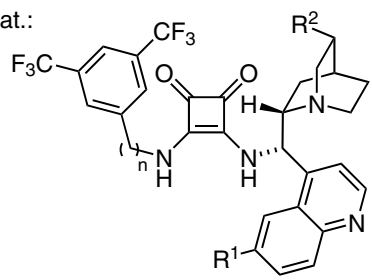

A: $n=1, R^{1}=O M e, R^{2}=$ vinyl

B: $n=0, R^{1}=O M e, R^{2}=$ vinyl

C: $n=0, R^{1}=H, R^{2}=$ vinyl

D: $n=1, R^{1}=O M e, R^{2}=E t$

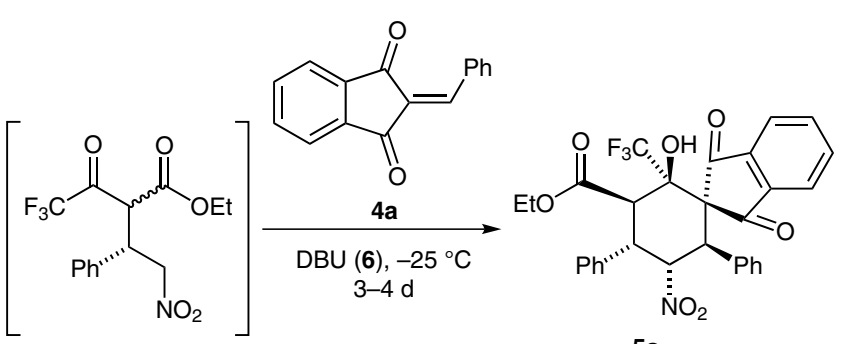

3a<smiles>[R]c1ccc2nccc(C(Nc3c(NN=Cc4cc(C(F)(F)F)cc(C(F)(F)F)c4)c(=O)c3=O)C3C4CCC3C(C=C)C4)c2c1</smiles><smiles>CC(C(NC(=S)Nc1cc(C(F)(F)F)cc(C(F)(F)F)c1)c1ccccc1)N1CCCCC1</smiles>

H

$F \cdot n=0, R=O N$

G: $n=0, R=H$

\begin{tabular}{|c|c|c|c|c|c|c|c|}
\hline Entry & Catalyst & Catalyst Loading (mol\%) & 6 (equiv) & $4 a$ (equiv) & Yield (\%) ${ }^{\mathrm{b}}$ & $\mathrm{dr}^{\mathrm{c}}$ & ee $(\%)^{d}$ \\
\hline 1 & A & 0.5 & 0.2 & 2.0 & 70 & $1: 1.1$ & 82 \\
\hline 2 & B & 0.5 & 0.2 & 2.0 & 62 & $1.1: 1$ & 91 \\
\hline 3 & C & 0.5 & 0.2 & 2.0 & 37 & $1.1: 1$ & 91 \\
\hline 4 & D & 0.5 & 0.2 & 2.0 & 63 & $1: 1$ & 88 \\
\hline 5 & E & 0.5 & 0.2 & 2.0 & 48 & $1.1: 1$ & $49^{e}$ \\
\hline 6 & $\mathbf{F}$ & 0.5 & 0.2 & 2.0 & 43 & $1.1: 1$ & $89^{e}$ \\
\hline 7 & G & 0.5 & 0.2 & 2.0 & 68 & $1: 1.1$ & $74^{e}$ \\
\hline 8 & H & 0.5 & 0.2 & 2.0 & 65 & $1.1: 1$ & 77 \\
\hline 9 & B & 5 & 0.2 & 2.0 & 46 & $1: 1$ & 94 \\
\hline 10 & B & 2.5 & 0.2 & 2.0 & 57 & $1.3: 1$ & 79 \\
\hline 11 & B & 1 & 0.2 & 2.0 & 58 & $1.2: 1$ & 86 \\
\hline 12 & C & 5 & 0.2 & 2.0 & 45 & $1: 1$ & 93 \\
\hline 13 & C & 2.5 & 0.2 & 2.0 & 51 & $1.2: 1$ & 91 \\
\hline 14 & C & 1 & 0.2 & 2.0 & 64 & $1.2: 1$ & 92 \\
\hline 15 & C & 1 & 0.1 & 2.0 & 48 & $1.4: 1$ & 93 \\
\hline 16 & C & 1 & 0.5 & 2.0 & 26 & $2.4: 1$ & 93 \\
\hline 17 & C & 1 & 0.2 & 1.5 & 59 & $1.5: 1$ & 93 \\
\hline 18 & C & 1 & 0.2 & 4.0 & 57 & $1: 1.1$ & 93 \\
\hline
\end{tabular}

${ }^{a}$ Reactions were carried out with $\mathbf{1 a}(0.5 \mathrm{mmol}), \mathbf{2 a}(0.5 \mathrm{mmol}, 1$ equiv), the indicated amount of catalyst, $4 \mathrm{a}$ ( $1.0 \mathrm{mmol}, 2$ equiv), and DBU (6; $0.1 \mathrm{mmol}, 0.2$ equiv) in $\mathrm{CH}_{2} \mathrm{Cl}_{2}(0.6 \mathrm{~mL}, \mathrm{C}=0.8 \mathrm{M})$.

${ }^{\mathrm{b}}$ Yield of the isolated product after column chromatography.

C Ratio of $(2 R, 3 R, 4 S, 5 R, 6 R)-5 \mathbf{a} /(2 S, 3 S, 4 S, 5 R, 6 R)-5$ a determined by ${ }^{1} \mathrm{H}$ NMR spectroscopy.

${ }^{d}$ Enantiomeric excess of the major diastereomer determined by HPLC on a chiral stationary phase.

e The opposite enantiomer is formed. 
Table 3 Substrate Scope of the Michael/Michael/Aldol One-Pot Reaction ${ }^{\mathrm{a}, 12}$

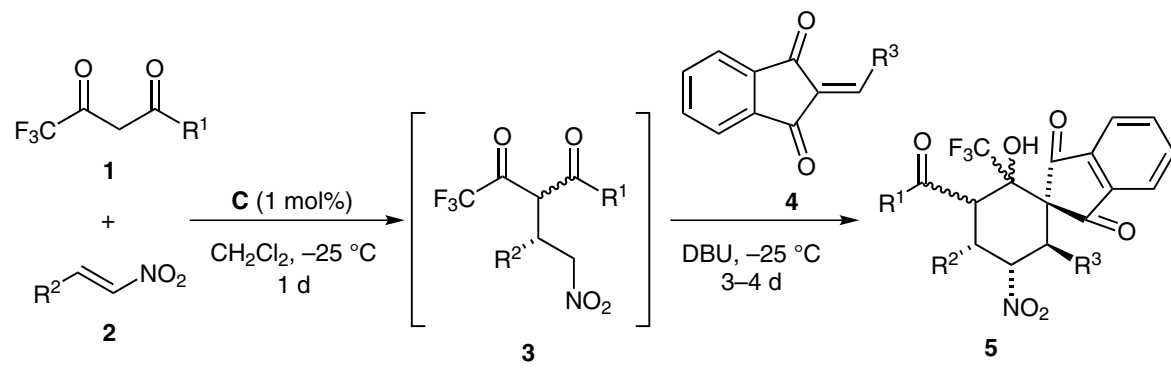

\begin{tabular}{|c|c|c|c|c|c|c|}
\hline 5 & $\mathrm{R}^{1}$ & $\mathrm{R}^{2}$ & $\mathrm{R}^{3}$ & Yield (\%) & $d r^{c}$ & ee $(\%)^{d}$ \\
\hline $\mathbf{a}$ & OEt & $\mathrm{Ph}$ & $\mathrm{Ph}$ & 64 & $1.2: 1$ & 92 \\
\hline $\mathbf{b}$ & OEt & $2-\mathrm{ClC}_{6} \mathrm{H}_{4}$ & $\mathrm{Ph}$ & 59 & $5: 1$ & $85(86)^{e}$ \\
\hline c & OEt & 4- $-\mathrm{FC}_{6} \mathrm{H}_{4}$ & $\mathrm{Ph}$ & 52 & $1: 1(>20: 1)$ & 88 \\
\hline d & OEt & 4- $\mathrm{MeC}_{6} \mathrm{H}_{4}$ & $\mathrm{Ph}$ & 60 & $1: 1$ & 82 \\
\hline e & OEt & 4- $\mathrm{MeOC}_{6} \mathrm{H}_{4}$ & $\mathrm{Ph}$ & 54 & $1.6: 1$ & 72 \\
\hline $\mathbf{f}$ & OEt & thien-2-yl & $\mathrm{Ph}$ & 68 & $7: 1 f$ & $89^{f}$ \\
\hline $\mathbf{g}$ & OEt & N-Boc-indol-3-yl & $\mathrm{Ph}$ & 54 & $1.5: 1(>20: 1)$ & $28^{\mathrm{e}, \mathrm{g}}$ \\
\hline $\mathbf{h}$ & OEt & naphth-1-yl & $\mathrm{Ph}$ & 47 & $20: 1$ & 89 \\
\hline $\mathbf{i}$ & OEt & $\mathrm{Ph}$ & $4-\mathrm{BrC}_{6} \mathrm{H}_{4}$ & 72 & $1.3: 1$ & $0^{\text {h }}$ \\
\hline $\mathbf{j}$ & OEt & $\mathrm{Ph}$ & $4-\mathrm{NO}_{2} \mathrm{C}_{6} \mathrm{H}_{4}$ & 23 & $1.5: 1(10: 1)$ & $81^{e}$ \\
\hline $\mathbf{k}$ & OEt & $\mathrm{Ph}$ & 3- $\mathrm{MeOC}_{6} \mathrm{H}_{4}$ & 47 & $1.4: 1$ & $92(91)$ \\
\hline I & OEt & $\mathrm{Ph}$ & 4- $\mathrm{MeC}_{6} \mathrm{H}_{4}$ & 35 & $1: 1$ & 75 \\
\hline $\mathbf{m}$ & OMe & $\mathrm{Ph}$ & $\mathrm{Ph}$ & 52 & $1: 1$ & $86(88)$ \\
\hline $\mathbf{n}$ & $\mathrm{Ph}$ & $\mathrm{Ph}$ & $\mathrm{Ph}$ & 54 & $1.3: 1$ & $0^{\mathrm{e}, \mathrm{h}}$ \\
\hline \multicolumn{7}{|c|}{ 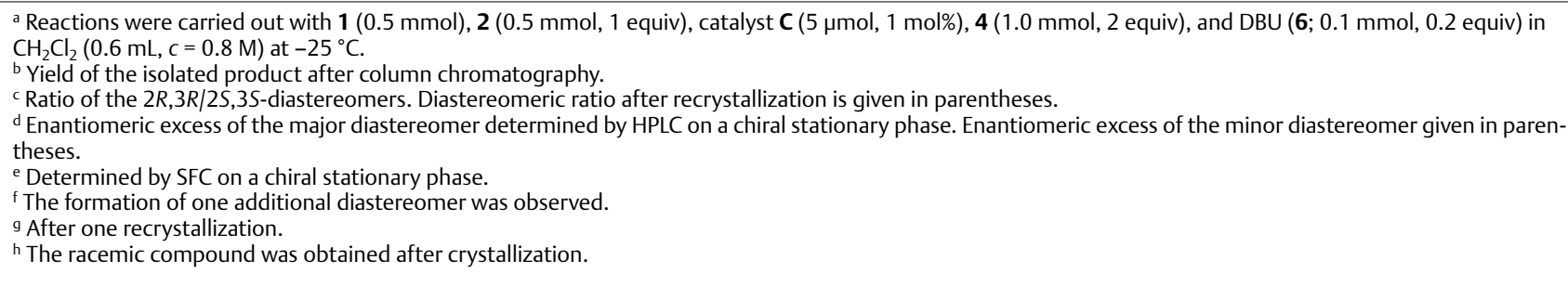 } \\
\hline
\end{tabular}

case of the p-methyl- 2d and p-methoxy-substituted nitrostyrene $\mathbf{2 e}$, the enantioselectivity dropped to $82 \%$ and $72 \%$, respectively. Furthermore, an aliphatic nitroalkene was tested, but no reaction occurred in this case. ${ }^{12}$ For the next set of alterations, the substituent $\mathrm{R}^{3}$ at the 2-arylideneindandione $\mathbf{4}$ was changed. We observed that an electron-deficient alkene (Table $3,5 i$ ) provides better results than electron-neutral or electron-rich Michael acceptors (Table 3, 5k, 1). In consequence, the strongly electron-withdrawing nature of the nitro and trifluoromethyl group should enhance the electrophilicity of the alkene $\mathbf{4}$, and therefore an increased reaction rate should be observed. To our surprise, the yield decreased to $23 \%$ when using the corresponding alkene $\mathbf{4 j}$ and in the case of the trifluoromethyl-substituted alkene the reaction did not work at all. Different heteroaro- matic substituents at $\mathbf{4}$ inhibited the reaction. For the last set of variations, our attention was focused on the 1,3-dicarbonyl substrate $\mathbf{1}$. Both, the methyl $\beta$-keto ester and the benzoyl-substituted trifluoroacetone were converted successfully into the corresponding cyclohexanes $\mathbf{5 m}$ and $\mathbf{5 n}$ in $52 \%$ and $54 \%$ yield and high enantioselectivity, albeit with a low diastereomeric ratio.

Finally, in order to demonstrate the preparative applicability of the new organocatalytic one-pot Michael/ Michael/aldol reaction, the protocol was examined on a gram-scale (Scheme 2). The relative and absolute configuration of the products $\mathbf{5}$ was determined by NOESY experiments and by X-ray crystal structure analysis of the representative compound $\mathbf{5 b}$ (Figure 2). ${ }^{13}$ 


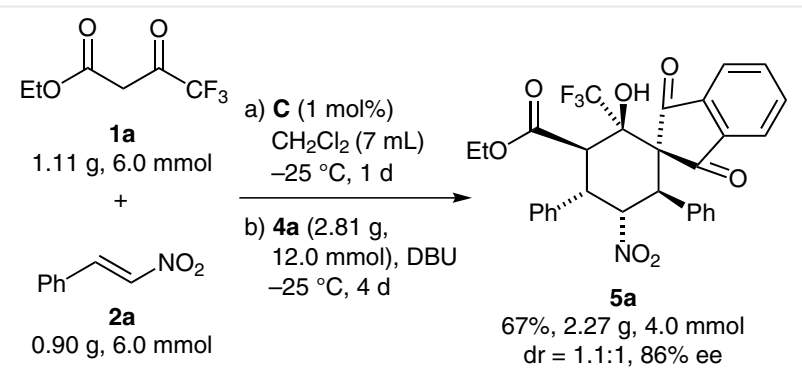

Scheme 2 Gram-scale one-pot synthesis of 5 a
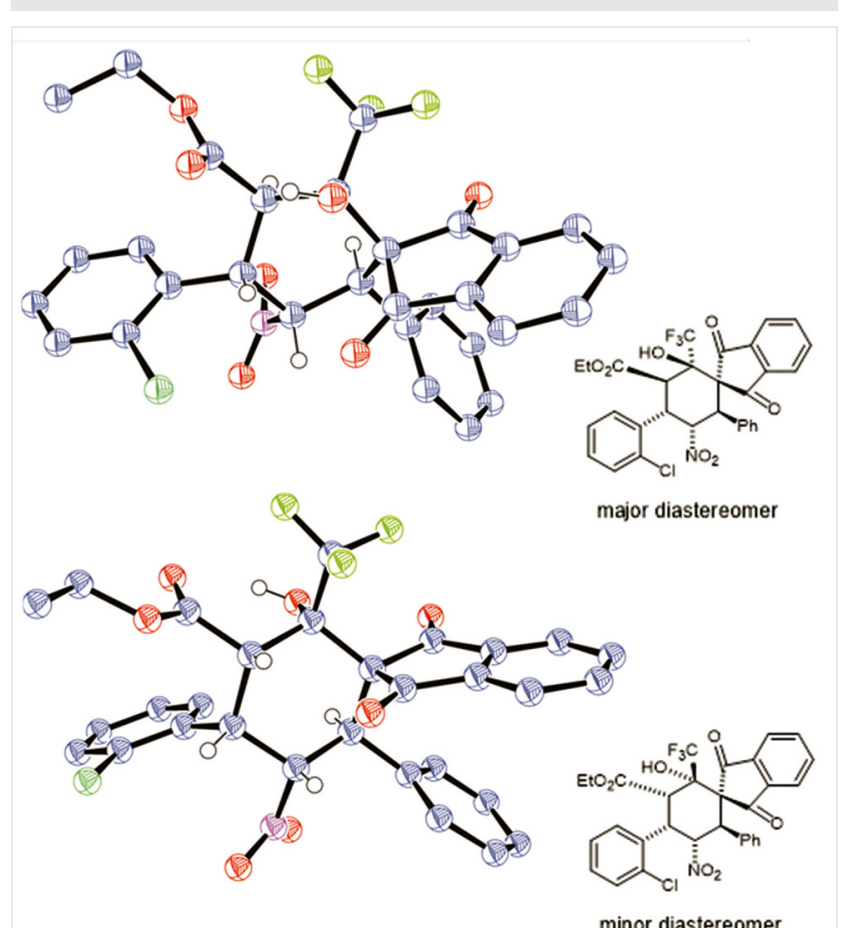

Figure 2 X-ray crystal structure of both diastereomers of $\mathbf{5 b}^{13}$

In conclusion, we have developed a new asymmetric one-pot Michael/Michael/aldol reaction leading to highly substituted spirocyclohexane indan-1,3-diones bearing five adjacent stereogenic centers including a trifluoromethylated tetrasubstituted one. Only $1 \mathrm{~mol} \%$ of a cinchona-derived squaramide catalyst was enough to obtain the desired compounds starting from trifluoromethyl-substituted 1,3-dicarbonyl compounds, nitroolefins, and arylidene indandiones in medium to very good yields and enantioselectivities, but generally low up to high diastereomeric ratios. Especially the combination of the trifluoromethyl substituent and the spiroindandione moiety renders the compounds interesting for medicinal chemistry.

Commercially available compounds were used without further purification. Solvents were distilled using standard procedures. Flash column chromatography was performed with silica gel SIL G-25 UV254 (size $0.040-0.063 \mathrm{~mm}$ ) from Machery \& Nagel. For the TLC silica gel 60 F254 plates from Merck, Darmstadt, were used. The compounds on the TLC plates were identified under UV light ( $254 \mathrm{~nm}$ ) and by staining with anisaldehyde staining reagent. ${ }^{1} \mathrm{H},{ }^{13} \mathrm{C}$, and ${ }^{19} \mathrm{~F}$ NMR spectra were recorded on with Varian Gemini 300, Varian Mercury 300, Varian Inova 400, and Varian Inova 600 instruments at ambient temperature. Signals of the diastereomer are marked with a superscript 'a'. Mass spectra were recorded with the spectrometer SSQ7000 from Finnigan at $70 \mathrm{eV}$, whereas HRMS data (ESI) were collected with a ThermoFisher Scientific LTQ-Orbitrap XL apparatus. The IR spectra were recorded on a PerkinElmer FT-IR Spectrum 100 using the ATR technique. Melting points were measured with a Büchi 510 melting point apparatus. For the determination of the enantiomeric excesses, the HPLC data were collected with either Hewlett-Packard 1050, Agilent 1100, or Agilent 1260 instruments using Chiracel (OD, OJ), Chiralpak (AD, AS, IA, IC) columns from Daicel. The analytical SFC was conducted with a SFC Method - Station II from Thar SFC Waters including a $(R, R)$-Whelk-01 column from Merck and Chiralpak IA column from Daicel. Some compounds seem to be impure due to critical instability in solution and under the applied chromatography conditions optical rotations were determined on a PerkinElmer P241 polarimeter.

\section{One-Pot Michael/Michael/Aldol Reaction; General Procedure}

A solution of nitroalkene $\mathbf{2}(0.5 \mathrm{mmol}, 1$ equiv), trifluoromethylated $\beta$-dicarbonyl compound 1 ( $0.5 \mathrm{mmol}, 1$ equiv), and squaramide catalyst $\mathbf{C}(0.05 \mathrm{mmol}, 1 \mathrm{~mol} \%)$ in $\mathrm{CH}_{2} \mathrm{Cl}_{2}(0.6 \mathrm{~mL})$ was stirred at $-25^{\circ} \mathrm{C}$ for $24 \mathrm{~h}$. Subsequently, the reaction mixture was treated with 2arylidene indandione $\mathbf{4}$ ( $1.0 \mathrm{mmol}, 2$ equiv) and $\mathrm{DBU}(\mathbf{6} ; 15.2 \mathrm{mg}, 0.1$ mmol, 0.2 equiv) and the stirring was continued at $-25^{\circ} \mathrm{C}$ for 3 days. The crude reaction mixture was purified by column chromatography (pentane- $\mathrm{Et}_{2} \mathrm{O}, 10: 1$ to 2:1) and recrystallization (Table 3).

Ethyl $(2 R, 3 R, 4 S, 5 R, 6 R)$-2-Hydroxy-5-nitro-1',3'-dioxo-4,6-diphenyl-2-(trifluoromethyl)-1',3'-dihydrospiro[cyclohexane-1,2'-indene]-3-carboxylate (5a)

Yield: $182 \mathrm{mg}(64 \%, \mathrm{dr}=1.2: 1)$, colorless solid; $\mathrm{mp} 98-100{ }^{\circ} \mathrm{C} ; 92 \%$ ee [HPLC: Chiralpak IC]; $R_{f}=0.29$ (n-pentane-Et $\left.{ }_{2} \mathrm{O}, 2: 1\right) ;[\alpha]_{\mathrm{D}}{ }^{22}-42.9(c=$ $\left.1.01, \mathrm{CHCl}_{3}\right)$.

IR (ATR): 3379, 2985, 2189, 2082, 2001, 1915, 1709, 1555, 1462, 1356, $1249,1185,1018,949,844,757,701 \mathrm{~cm}^{-1}$.

${ }^{1} \mathrm{H}$ NMR $\left(600 \mathrm{MHz}, \mathrm{CDCl}_{3}\right): \delta=7.97\left(\mathrm{~d},{ }^{3} \mathrm{~J}=7.4 \mathrm{~Hz}, 1 \mathrm{H}, \mathrm{ArH}\right), 7.86-$ $7.71(\mathrm{~m}, 3 \mathrm{H}, \mathrm{ArH}), 7.43-7.37(\mathrm{~m}, 2 \mathrm{H}, \mathrm{ArH}), 7.36-7.28(\mathrm{~m}, 3 \mathrm{H}, \mathrm{ArH})$, 7.11-7.00 (m, $5 \mathrm{H}, \mathrm{ArH}), 6.19(\mathrm{~s}, 1 \mathrm{H}, \mathrm{OH}), 5.95\left(\mathrm{dd},{ }^{3} \mathrm{~J}=11.1 \mathrm{~Hz}\right.$, $\left.{ }^{3} \mathrm{~J}=8.2 \mathrm{~Hz}, 1 \mathrm{H}, \mathrm{CHNO}_{2}\right), 4.84\left(\mathrm{dd},{ }^{3} \mathrm{~J}=12.8 \mathrm{~Hz},{ }^{3} \mathrm{~J}=8.2 \mathrm{~Hz}, 1 \mathrm{H}, \mathrm{PhCH}\right)$, $4.46\left(\mathrm{~d},{ }^{3} \mathrm{~J}=11.1 \mathrm{~Hz}, 1 \mathrm{H}, \mathrm{PhCH}\right), 4.28\left(\mathrm{~d},{ }^{3} \mathrm{~J}=12.8 \mathrm{~Hz} 1 \mathrm{H}, \mathrm{CHCO}_{2} \mathrm{Et}\right.$ ), 3.99-3.89 (m, $\left.1 \mathrm{H}, \mathrm{CH}_{3} \mathrm{CH}_{2}\right), 3.88-3.78\left(\mathrm{~m}, 1 \mathrm{H}, \mathrm{CH}_{3} \mathrm{CH}_{2}\right), 1.03(\mathrm{t}$, $\left.3 \mathrm{~J}=7.2 \mathrm{~Hz}, 3 \mathrm{H}, \mathrm{CH}_{3} \mathrm{CH}_{2}\right)$.

${ }^{13} \mathrm{C}$ NMR (101 MHz, $\mathrm{CDCl}_{3}$, C,F-decoupled): $\delta=198.2,195.6,172.8$, 143.3, 143.1, 136.3, 136.2, 133.9, 133.7, 129.6, 129.3, 129.1, 128.9, $128.8,128.6,124.8,123.7,123.5,91.7,79.3,62.6,62.4,46.5,43.3$, 42.6, 13.5 .

${ }^{19} \mathrm{~F}$ NMR (376 MHz, $\mathrm{CDCl}_{3}$ ): $\delta=-73.87$ (s, $3 \mathrm{~F}$ ).

MS (EI, $70 \mathrm{eV}): m / z=521\left(61,\left[\mathrm{M}-\mathrm{NO}_{2}\right]^{+}\right), 475\left(37,\left[\mathrm{M}-\mathrm{NO}_{2}-\mathrm{EtOH}\right]^{+}\right)$, 457 (35, [M - $\left.\left.\mathrm{NO}_{2}-\mathrm{EtOH}-\mathrm{H}_{2} \mathrm{O}\right]^{+}\right), 429$ (26), 365 (13), 279 (22), 233 (100), 205 (14), 191 (13), 189 (18), 173 (11), 149 (13), 115 (12), 104 (12), $91\left(16,\left[\mathrm{C}_{7} \mathrm{H}_{7}\right]^{+}\right), 77\left(9,\left[\mathrm{C}_{6} \mathrm{H}_{5}\right]^{+}\right)$.

MS (CI, methane): $m / z=596\left(4,[\mathrm{M}+\mathrm{Et}]^{+}\right), 568\left(44,[\mathrm{M}+\mathrm{H}]^{+}\right)$.

HRMS (ESI): $m / z[\mathrm{M}+\mathrm{Na}]^{+}$calcd for $\mathrm{C}_{30} \mathrm{H}_{24} \mathrm{~F}_{3} \mathrm{NO}_{7} \mathrm{Na}^{+}$: 590.1397; found: 590.1391 . 
Ethyl (2R,3R,4S,5R,6R)-4-(2-Chlorophenyl)-2-hydroxy-5-nitro1',3'-dioxo-6-phenyl-2-(trifluoromethyl)-1',3'-dihydrospiro[cyclohexane-1,2'-indene]-3-carboxylate ( $5 \mathbf{b})$

Yield: $178 \mathrm{mg}(59 \%, \mathrm{dr}=5: 1)$; colorless solid; $\mathrm{mp}>180^{\circ} \mathrm{C}$; $85 \%$ ee [SFC: $(R, R)-$ Whelk-01]; $R_{f}=0.26\left(n\right.$-pentane- $\left.\mathrm{Et}_{2} \mathrm{O}, 2: 1\right) ;[\alpha]_{\mathrm{D}}^{22}-56.3$ ( $c=0.81, \mathrm{CHCl}_{3}$ ).

IR (ATR): 3401, 2984, 2637, 2221, 2085, 2001, 1709, 1554, 1465, 1352 , $1252,1185,1020,951,848,753,704 \mathrm{~cm}^{-1}$.

${ }^{1} \mathrm{H}$ NMR $\left(600 \mathrm{MHz}, \mathrm{CDCl}_{3}\right): \delta=8.04\left(\mathrm{~d},{ }^{3} \mathrm{~J}=7.9 \mathrm{~Hz}, 1 \mathrm{H}, \mathrm{ArH}\right), 7.84-$ $7.75(\mathrm{~m}, 2 \mathrm{H}, \mathrm{ArH}), 7.74-7.67(\mathrm{~m}, 1 \mathrm{H}, \mathrm{ArH}), 7.44\left(\mathrm{dd},{ }^{3} \mathrm{~J}=7.7 \mathrm{~Hz}\right.$, $\left.{ }^{4} J=1.2 \mathrm{~Hz}, 1 \mathrm{H}, \mathrm{ArH}\right), 7.33\left(\mathrm{dd},{ }^{3} J=7.9 \mathrm{~Hz},{ }^{4} J=1.5 \mathrm{~Hz}, 1 \mathrm{H}, \mathrm{ArH}\right), 7.28-$ 7.16 (m, $2 \mathrm{H}, \mathrm{ArH}), 7.14-7.01(\mathrm{~m}, 5 \mathrm{H}, \mathrm{ArH}), 6.05-5.91\left(\mathrm{~m}, 2 \mathrm{H}, \mathrm{CHNO}_{2}\right.$, $\mathrm{OH}), 5.52\left(\mathrm{dd},{ }^{3} \mathrm{~J}=12.9 \mathrm{~Hz},{ }^{3} \mathrm{~J}=7.9 \mathrm{~Hz}, 1 \mathrm{H}, 2-\mathrm{ClC}_{6} \mathrm{H}_{4} \mathrm{CH}\right), 4.45(\mathrm{~d}$, $\left.{ }_{3}^{3}=10.9 \mathrm{~Hz}, 1 \mathrm{H}, \mathrm{PhCH}\right), 4.29\left(\mathrm{~d},{ }^{3} \mathrm{~J}=12.9 \mathrm{~Hz}, 1 \mathrm{H}, \mathrm{CHCO}_{2} \mathrm{Et}\right), 4.04-3.94$ $\left(\mathrm{m}, 1 \mathrm{H}, \mathrm{CH}_{3} \mathrm{CH}_{2}\right), 3.94-3.84\left(\mathrm{~m}, 1 \mathrm{H}, \mathrm{CH}_{3} \mathrm{CH}_{2}\right), 1.08\left(\mathrm{t},{ }^{3} \mathrm{~J}=7.2 \mathrm{~Hz}, 3 \mathrm{H}\right.$, $\left.\mathrm{CH}_{3} \mathrm{CH}_{2}\right)$.

${ }^{13} \mathrm{C}$ NMR (101 MHz, $\mathrm{CDCl}_{3}$, C,F-decoupled): $\delta=197.4,195.3,172.9$, 143.5, 143.2, 136.3, 136.1, 135.8, 133.8, 131.9, 130.1, 130.1, 129.8, 129.0, 128.9, 128.7, 126.9, 125.4, 123.6, 123.6, 90.6, 79.2, 62.9, 62.8, 46.1, 42.9, 38.1, 13.6 .

${ }^{19} \mathrm{~F} \operatorname{NMR}\left(376 \mathrm{MHz}, \mathrm{CDCl}_{3}\right): \delta=-73.9(\mathrm{~s}, 3 \mathrm{~F})$.

MS (EI, $70 \mathrm{eV}): m / z=566\left(8,[\mathrm{M}-\mathrm{Cl}]^{+}\right), 555\left(100,\left[\mathrm{M}-\mathrm{NO}_{2}\right]^{+}\right), 509(16$, $\left.\left[\mathrm{M}-\mathrm{NO}_{2}-\mathrm{EtOH}\right]^{+}\right), 491\left(75,\left[\mathrm{M}-\mathrm{NO}_{2}-\mathrm{EtOH}-\mathrm{H}_{2} \mathrm{O}\right]^{+}\right), 463(43), 399$ (30), 313 (11), 267 (18), 233 (34), 149 (16), 91 (11, [ $\left.\left.\mathrm{C}_{7} \mathrm{H}_{7}\right]^{+}\right), 77$ (6, $\left.\left[\mathrm{C}_{6} \mathrm{H}_{5}\right]^{+}\right)$.

MS (CI, methane): $m / z=630\left(5,[\mathrm{M}+\mathrm{Et}]^{+}\right), 602\left(26,[\mathrm{M}+\mathrm{H}]^{+}\right)$.

HRMS (ESI): $m / z[\mathrm{M}+\mathrm{Na}]^{+}$calcd for $\mathrm{C}_{30} \mathrm{H}_{23}{ }^{35} \mathrm{ClF}_{3} \mathrm{NO}_{7} \mathrm{Na}^{+}: 624.1007$; found: 624.1009.

Ethyl (2S,3S,4S,5R,6R)-4-(4-Fluorophenyl)-2-hydroxy-5-nitro1',3'-dioxo-6-phenyl-2-(trifluoromethyl)-1',3'-dihydrospiro[cyclohexane-1,2'-indene]-3-carboxylate (5c)

Yield: $155 \mathrm{mg}$ ( $52 \%, \mathrm{dr}=1: 1)$; colorless solid; 88\% ee [HPLC: Chiralpak IC]; $R_{f}=0.27$ ( $n$-pentane-Et ${ }_{2} \mathrm{O}, 2: 1$ ).

IR (ATR): 3785, 3347, 2987, 2639, 2291, 2174, 2056, 1988, 1904, 1709, $1552,1365,1176,1021,850,765,704 \mathrm{~cm}^{-1}$.

${ }^{1} \mathrm{H}$ NMR $\left(600 \mathrm{MHz}, \mathrm{CDCl}_{3}\right): \delta=7.92-7.80(\mathrm{~m}, 1 \mathrm{H}, \mathrm{ArH}), 7.77-7.55(\mathrm{~m}$, $5 \mathrm{H}, \mathrm{ArH}), 7.24-6.69(\mathrm{~m}, 7 \mathrm{H}, \mathrm{ArH}), 6.43\left(\mathrm{dd},{ }^{3} \mathrm{~J}=13.1 \mathrm{~Hz},{ }^{3} \mathrm{~J}=7.7 \mathrm{~Hz}\right.$, $\left.1 \mathrm{H}, \mathrm{CHNO}_{2}\right), 6.06(\mathrm{~s}, 1 \mathrm{H}, \mathrm{OH}), 4.92\left(\mathrm{~d},{ }^{3} \mathrm{~J}=13.1 \mathrm{~Hz}, 1 \mathrm{H}, \mathrm{PhCH}\right), 4.58$ ('t', $\left.{ }^{3} \mathrm{~J}=7.7 \mathrm{~Hz}, 1 \mathrm{H}, 4-\mathrm{FC}_{6} \mathrm{H}_{4} \mathrm{CH}\right), 4.19\left(\mathrm{~d},{ }^{3} \mathrm{~J}=7.7 \mathrm{~Hz}, 1 \mathrm{H}, \mathrm{CHCO}_{2} \mathrm{Et}\right)$, 4.04-3.88 (m, $\left.2 \mathrm{H}, \mathrm{CH}_{3} \mathrm{CH}_{2}\right), 1.16\left(\mathrm{t},{ }^{3} \mathrm{~J}=7.2 \mathrm{~Hz}, 3 \mathrm{H}, \mathrm{CH}_{3} \mathrm{CH}_{2}\right)$.

${ }^{13} \mathrm{C}$ NMR (101 MHz, $\mathrm{CDCl}_{3}, \mathrm{C}, \mathrm{F}-$ decoupled): $\delta=199.2,195.1,170.7$, 163.2, 142.5, 141.3, 136.6, 135.9, 133.1, 132.8, 129.2, 128.5, 124.7, 123.5, 123.2, 115.7, 84.1, 78.2, 62.5, 58.8, 45.9, 44.0, 42.7, 14.0.

${ }^{19} \mathrm{~F} \mathrm{NMR}\left(376 \mathrm{MHz}, \mathrm{CDCl}_{3}\right): \delta=-72.0(\mathrm{~s}, 3 \mathrm{~F}),-112.5(\mathrm{~m}, 1 \mathrm{~F})$.

MS (EI, $70 \mathrm{eV}): m / z=539\left(27,\left[\mathrm{M}-\mathrm{NO}_{2}\right]^{+}\right), 493\left(17,\left[\mathrm{M}-\mathrm{NO}_{2}-\mathrm{EtOH}\right]^{+}\right)$, $475\left(21,\left[\mathrm{M}-\mathrm{NO}_{2}-\mathrm{EtOH}-\mathrm{H}_{2} \mathrm{O}\right]^{+}\right), 447$ (12), 251 (100), 233 (98), 223 (21), 209 (21), 207 (18), 202 (13), 173 (48), 149 (45), 133 (36), 120 (3), $115(40), 109\left(73,\left[\mathrm{C}_{7} \mathrm{H}_{6} \mathrm{~F}\right]^{+}\right), 104(50), 101(47), 91\left(60,\left[\mathrm{C}_{7} \mathrm{H}_{7}\right]^{+}\right), 77$ $\left(25,\left[\mathrm{C}_{6} \mathrm{H}_{5}\right]^{+}\right), 76\left(51,\left[\mathrm{C}_{6} \mathrm{H}_{4}\right]^{+}\right), 69\left(41,\left[\mathrm{CF}_{3}\right]^{+}\right)$.

MS (CI, methane): $m / z=614\left(2,[\mathrm{M}+\mathrm{Et}]^{+}\right), 586\left(100,[\mathrm{M}+\mathrm{H}]^{+}\right)$.

HRMS (ESI): $m / z[\mathrm{M}+\mathrm{Na}]^{+}$calcd for $\mathrm{C}_{30} \mathrm{H}_{23} \mathrm{~F}_{4} \mathrm{NO}_{7} \mathrm{Na}^{+}$: 608.1303; found: 608.1306 .
Ethyl (2R,3R,4S,5R,6R)-2-Hydroxy-5-nitro-1',3'-dioxo-6-phenyl-4(p-tolyl)-2-(trifluoromethyl)-1',3'-dihydrospiro[cyclohexane-1,2'indene]-3-carboxylate (5d)

Yield: $176 \mathrm{mg}(60 \%, \mathrm{dr}=1: 1)$; colorless solid; $82 \%$ ee [HPLC: Chiralpak IC]; $R_{f}=0.23$ ( $n$-pentane-Et ${ }_{2} \mathrm{O}, 2: 1$ ).

IR (ATR): 3410, 2984, 2310, 2037, 1900, 1708, 1555, 1455, 1350, 1248 , $1177,1021,847,770,702 \mathrm{~cm}^{-1}$.

${ }^{1} \mathrm{H}$ NMR $\left(600 \mathrm{MHz}, \mathrm{CDCl}_{3}\right): \delta$ (mixture of diastereomers) $=7.95(\mathrm{~d}$, $\left.{ }^{3} \mathrm{~J}=7.4 \mathrm{~Hz}, 1 \mathrm{H}, \mathrm{ArH}\right), 7.88-7.60(\mathrm{~m}, 7 \mathrm{H}, \mathrm{ArH}), 7.59-7.47(\mathrm{~m}, 1 \mathrm{H}$, ArH), 7.33-7.24 (m, $3 \mathrm{H}, \mathrm{ArH}), 7.12\left(\mathrm{~d},{ }^{3} \mathrm{~J}=7.4 \mathrm{~Hz}, 5 \mathrm{H}, \mathrm{ArH}\right), 7.08-7.00$ $(\mathrm{m}, 6 \mathrm{H}, \mathrm{ArH}), 6.90\left(\mathrm{t},{ }^{3} \mathrm{~J}=7.2 \mathrm{~Hz}, 3 \mathrm{H}, \mathrm{ArH}\right), 6.44^{\mathrm{a}}\left(\mathrm{dd},{ }^{3} \mathrm{~J}=12.9,{ }^{3} \mathrm{~J}=7.7\right.$ $\left.\mathrm{Hz}, 1 \mathrm{H}, \mathrm{CHNO}_{2}\right), 6.18(\mathrm{~s}, 1 \mathrm{H}, \mathrm{OH}), 6.09^{\mathrm{a}}(\mathrm{s}, 1 \mathrm{H}, \mathrm{OH}), 5.93\left(\mathrm{dd},{ }^{3} \mathrm{~J}=11.1\right.$ $\left.\mathrm{Hz},{ }^{3} \mathrm{~J}=8.2 \mathrm{~Hz}, 1 \mathrm{H}, \mathrm{CHNO}_{2}\right), 4.99^{\mathrm{a}}\left(\mathrm{d},{ }^{3} \mathrm{~J}=12.9 \mathrm{~Hz}, 1 \mathrm{H}, \mathrm{PhCH}\right), 4.80$ (dd, $\left.{ }^{3} J=12.6 \mathrm{~Hz},{ }^{3} \mathrm{~J}=8.2 \mathrm{~Hz}, 1 \mathrm{H}, p-\mathrm{TolCH}\right), 4.55^{\mathrm{a}}$ ('t', $3 \mathrm{~J}=7.7 \mathrm{~Hz}, 1 \mathrm{H}, p-$ TolCH), 4.27 (d, $\left.{ }^{3} \mathrm{~J}=12.6 \mathrm{~Hz}, 1 \mathrm{H}, \mathrm{CHCO}_{2} \mathrm{Et}\right), 4.45\left(\mathrm{~d},{ }^{3} \mathrm{~J}=11.1 \mathrm{~Hz}, 1 \mathrm{H}\right.$, $\mathrm{PhCH}), 4.19^{\mathrm{a}}\left(\mathrm{d},{ }^{3} \mathrm{~J}=7.7 \mathrm{~Hz}, 1 \mathrm{H}, \mathrm{CHCO}_{2} \mathrm{Et}\right), 4.02-3.78\left(\mathrm{~m}, 4 \mathrm{H}, \mathrm{CH}_{3} \mathrm{CH}_{2}\right)$, $2.30\left(\mathrm{~s}, 6 \mathrm{H}, \mathrm{Ar}-\mathrm{CH}_{3}\right), 1.16\left(\mathrm{t},{ }^{3} \mathrm{~J}=7.2 \mathrm{~Hz}, 3 \mathrm{H}, \mathrm{CH}_{3} \mathrm{CH}_{2}\right), 1.05\left(\mathrm{t},{ }^{3} \mathrm{~J}=7.2\right.$ $\left.\mathrm{Hz}, 3 \mathrm{H}, \mathrm{CH}_{3} \mathrm{CH}_{2}\right)$.

${ }^{13} \mathrm{C}$ NMR (101 MHz, $\mathrm{CDCl}_{3}$, C,F-decoupled): $\delta$ (mixture of diastereomers $)=199.3,198.2,195.6,195.0,172.9,170.9^{a}, 143.3,143.1,142.8$, $141.3,139.0,138.8,136.5,136.3,136.2,135.7,134.0,133.1,131.1$, 130.6, 130.3, 129.5, 129.4, 128.9, 128.6, 128.4, 126.1, 124.2, 123.7, $123.5,123.2,91.8,84.3^{\mathrm{a}}, 79.3,78.2^{\mathrm{a}}, 62.5,62.5,62.4^{\mathrm{a}}, 58.9,46.4,46.4^{\mathrm{a}}$, $44.0^{\mathrm{a}}, 43.3,42.9^{\mathrm{a}}, 42.2,21.3,14.0,13.6$.

$\left.{ }^{19} \mathrm{~F} \mathrm{NMR} \mathrm{(376} \mathrm{MHz,} \mathrm{CDCl}_{3}\right): \delta$ (mixture of diastereomers) $=-72.1(\mathrm{~s}, 3$ F), $-73.9(\mathrm{~s}, 3 \mathrm{~F})$.

MS (EI, $70 \mathrm{eV}): m / z=535\left(38,\left[\mathrm{M}-\mathrm{NO}_{2}\right]^{+}\right), 489\left(30,\left[\mathrm{M}-\mathrm{NO}_{2}-\mathrm{EtOH}\right]^{+}\right)$, $471\left(23,\left[\mathrm{M}-\mathrm{NO}_{2}-\mathrm{EtOH}-\mathrm{H}_{2} \mathrm{O}\right]^{+}\right), 443(13), 293$ (32), 247 (51), 233 (100), 205 (19), 203 (44), 178 (13), 149 (13), 145 (10), 129 (12), 117 (12), 115 (25), 105 (19), $91\left(16,\left[\mathrm{C}_{7} \mathrm{H}_{7}\right]^{+}\right), 77\left(7,\left[\mathrm{C}_{6} \mathrm{H}_{5}\right]^{+}\right), 76(14$, $\left.\left[\mathrm{C}_{6} \mathrm{H}_{4}\right]^{+}\right)$.

MS (CI, methane): $m / z=610\left(7,[\mathrm{M}+\mathrm{Et}]^{+}\right), 582\left(48,[\mathrm{M}+\mathrm{H}]^{+}\right)$.

HRMS (ESI): $m / z[\mathrm{M}+\mathrm{Na}]^{+}$calcd for $\mathrm{C}_{31} \mathrm{H}_{26} \mathrm{~F}_{3} \mathrm{NO}_{7} \mathrm{Na}^{+}$: 604.1554; found: 604.1553 .

Ethyl (2R,3R,4S,5R,6R)-2-Hydroxy-4-(4-methoxyphenyl)-5-nitro1',3'-dioxo-6-phenyl-2-(trifluoromethyl)-1',3'-dihydrospiro[cyclohexane-1,2'-indene]-3-carboxylate (5e)

Yield: $160 \mathrm{mg}(54 \%, \mathrm{dr}=1.6: 1)$; colorless solid; $72 \%$ ee [HPLC: Chiralpak IC]; $R_{f}=0.20$ ( $n$-pentane- $\mathrm{Et}_{2} \mathrm{O}, 2: 1$ ).

IR (ATR): 3370, 2963, 2312, 2078, 1894, 1704, 1555, 1457, 1350, 1254, $1181,1023,933,847,765,703 \mathrm{~cm}^{-1}$.

${ }^{1} \mathrm{H}$ NMR $\left(600 \mathrm{MHz}, \mathrm{CDCl}_{3}\right): \delta$ (mixture of diastereomers $)=7.96(\mathrm{~d}$, $\left.{ }^{3} J=7.4 \mathrm{~Hz}, 1 \mathrm{H}, \mathrm{ArH}\right), 7.86-7.77(\mathrm{~m}, 3 \mathrm{H}, \mathrm{ArH}), 7.76-7.61(\mathrm{~m}, 4 \mathrm{H}$, ArH), 7.32 (d, $\left.{ }^{3} \mathrm{~J}=8.9 \mathrm{~Hz}, 2 \mathrm{H}, \mathrm{ArH}\right), 7.17$ (br s, $\left.1 \mathrm{H}, \mathrm{ArH}\right), 7.09-7.00(\mathrm{~m}$, $5 \mathrm{H}, \mathrm{ArH}), 6.90\left(\mathrm{t},{ }^{3} \mathrm{~J}=7.2 \mathrm{~Hz}, 1 \mathrm{H}, \mathrm{ArH}\right), 6.84\left(\mathrm{~d},{ }^{3} \mathrm{~J}=8.9 \mathrm{~Hz}, 4 \mathrm{H}, \mathrm{ArH}\right)$, $6.43\left(\mathrm{dd},{ }^{3} \mathrm{~J}=12.9 \mathrm{~Hz},{ }^{3} \mathrm{~J}=7.6 \mathrm{~Hz}, 1 \mathrm{H}, \mathrm{CHNO}_{2}\right), 6.12^{\mathrm{a}}(\mathrm{s}, 0.5 \mathrm{H}, \mathrm{OH})$, $6.21(\mathrm{~s}, 1 \mathrm{H}, \mathrm{OH}), 5.92\left(\mathrm{dd},{ }^{3} \mathrm{~J}=11.1 \mathrm{~Hz},{ }^{3} \mathrm{~J}=8.2 \mathrm{~Hz}, 1 \mathrm{H}, \mathrm{CHNO}_{2}\right), 4.96^{\mathrm{a}}$ $\left(\mathrm{d},{ }^{3} \mathrm{~J}=12.9 \mathrm{~Hz}, 0.5 \mathrm{H}, \mathrm{PhCH}\right), 4.78\left(\mathrm{dd},{ }^{3} \mathrm{~J}=12.6 \mathrm{~Hz},{ }^{3} \mathrm{~J}=8.2 \mathrm{~Hz}, 1 \mathrm{H}, 4-\right.$ $\mathrm{MeOC}_{6} \mathrm{H}_{4} \mathrm{CH}$ ), 4.54 ('t', ${ }^{3} \mathrm{~J}=7.6 \mathrm{~Hz}, 0.5 \mathrm{H}, 4-\mathrm{MeOC}_{6} \mathrm{H}_{4} \mathrm{CH}$ ), 4.44 (d, $\left.{ }^{3} J=11.1 \mathrm{~Hz}, 1 \mathrm{H}, \mathrm{PhCH}\right), 4.24\left(\mathrm{~d},{ }^{3} \mathrm{~J}=12.6 \mathrm{~Hz}, 1 \mathrm{H}, \mathrm{CHCO}_{2} \mathrm{Et}\right), 4.18^{\mathrm{a}}(\mathrm{d}$, $\left.{ }_{3}^{3}=7.6 \mathrm{~Hz}, 0.5 \mathrm{H}, \mathrm{CHCO}_{2} \mathrm{Et}\right), 4.02-3.81\left(\mathrm{~m}, 3 \mathrm{H}, \mathrm{CH}_{3} \mathrm{CH}_{2}\right), 3.80-3.75(\mathrm{~m}$, $\left.4.5 \mathrm{H}, \mathrm{OCH}_{3}\right), 1.17^{\mathrm{a}}\left(\mathrm{t},{ }^{3} \mathrm{~J}=7.2 \mathrm{~Hz}, 1.5 \mathrm{H}, \mathrm{CH}_{3} \mathrm{CH}_{2}\right), 1.05\left(\mathrm{t},{ }^{3} \mathrm{~J}=6.9 \mathrm{~Hz}\right.$, $\left.3 \mathrm{H}, \mathrm{CH}_{3} \mathrm{CH}_{2}\right)$.

${ }^{13} \mathrm{C}$ NMR (101 MHz, $\mathrm{CDCl}_{3}$, C,F-decoupled): $\delta$ (mixture of diastereomers $)=199.3^{\mathrm{a}}, 198.2,195.6,195.0^{\mathrm{a}}, 172.9,170.9^{\mathrm{a}}, 160.0^{\mathrm{a}}, 160.0$, $143.3,143.0,142.6,141.3,136.5,136.3,136.2,135.7,134.0,133.0$, 
132.4, 130.5, 129.5, 128.9, 128.6, 128.4, 125.5, 125.1, 123.6, 123.5, $123.2,114.2,114.0,91.8,84.3^{\mathrm{a}}, 79.3,78.2^{\mathrm{a}}, 62.5,62.4^{\mathrm{a}}, 62.4,58.9^{\mathrm{a}}$, $55.3,55.2^{\mathrm{a}}, 46.4,46.0^{\mathrm{a}}, 44.0^{\mathrm{a}}, 43.5,42.8^{\mathrm{a}}, 41.9,14.0^{\mathrm{a}}, 13.6$.

$\left.{ }^{19} \mathrm{~F} \mathrm{NMR} \mathrm{(376} \mathrm{MHz,} \mathrm{CDCl}_{3}\right): \delta$ (mixture of diastereomers) $=-72.0(\mathrm{~s}, 3$ F), $-73.9(\mathrm{~s}, 3 \mathrm{~F})$.

MS (EI, $70 \mathrm{eV}): m / z=551\left(2,\left[\mathrm{M}-\mathrm{NO}_{2}\right]^{+}\right), 505\left(2,\left[\mathrm{M}-\mathrm{NO}_{2}-\mathrm{EtOH}\right]^{+}\right)$, 487 (1, [M - $\left.\mathrm{NO}_{2}-\mathrm{EtOH}-\mathrm{H}_{2} \mathrm{O}\right]^{+}$), 363 (7), 316 (6), 233 (100), 219 (15), 205 (5), 179 (18), 176 (8), 139 (5), 132 (11), 115 (7), 104 (5), 91 $\left(2,\left[\mathrm{C}_{7} \mathrm{H}_{7}\right]^{+}\right), 77\left(4,\left[\mathrm{C}_{6} \mathrm{H}_{5}\right]^{+}\right), 76\left(9,\left[\mathrm{C}_{6} \mathrm{H}_{4}\right]^{+}\right)$.

MS (CI, methane): $m / z=626\left(2,[\mathrm{M}+\mathrm{Et}]^{+}\right), 598\left(23,[\mathrm{M}+\mathrm{H}]^{+}\right)$.

HRMS (ESI): $m / z[\mathrm{M}+\mathrm{Na}]^{+}$calcd for $\mathrm{C}_{31} \mathrm{H}_{26} \mathrm{~F}_{3} \mathrm{NO}_{8} \mathrm{Na}^{+}$: 620.1503; found: 620.1502 .

\section{Ethyl (2S,3S,4R,5S,6R)-2-Hydroxy-5-nitro-1',3'-dioxo-6-phenyl-4- (thiophen-2-yl)-2-(trifluoromethyl)-1',3'-dihydrospiro[cyclohex- ane-1,2'-indene]-3-carboxylate (5f)}

Yield: $194 \mathrm{mg}(68 \%, \mathrm{dr}=7: 1)$; colorless solid; $89 \%$ ee [SFC: $(R, R)$ Whelk-01]; $R_{f}=0.20$ (n-pentane- $\mathrm{Et}_{2} \mathrm{O}, 2: 1$ ).

IR (ATR): 3401, 2995, 2626, 2229, 2088, 1997, 1702, 1560, 1452, 1347 , 1254, 1184, 1023, 849, 759, $703 \mathrm{~cm}^{-1}$.

${ }^{1} \mathrm{H}$ NMR $\left(600 \mathrm{MHz}, \mathrm{CDCl}_{3}\right): \delta=7.83\left(\mathrm{~d},{ }^{3} \mathrm{~J}=7.4 \mathrm{~Hz}, 1 \mathrm{H}, \mathrm{ArH}\right), 7.71-$ $7.59(\mathrm{~m}, 3 \mathrm{H}, \mathrm{ArH}), 7.32\left(\mathrm{~d},{ }^{3} \mathrm{~J}=5.0 \mathrm{~Hz}, 1 \mathrm{H}, \mathrm{ArH}\right), 7.22(\mathrm{br} \mathrm{s}, 1 \mathrm{H}, \mathrm{ArH})$, 7.04 (br s, $1 \mathrm{H}, \mathrm{ArH}), 6.99-6.77(\mathrm{~m}, 5 \mathrm{H}, \mathrm{ArH}), 6.43\left(\mathrm{dd},{ }^{3} \mathrm{~J}=12.9 \mathrm{~Hz}\right.$, $\left.{ }^{3} \mathrm{~J}=6.9 \mathrm{~Hz}, 1 \mathrm{H}, \mathrm{CHNO}_{2}\right), 6.01(\mathrm{~s}, 1 \mathrm{H}, \mathrm{OH}), 4.98-4.85(\mathrm{~m}, 2 \mathrm{H}$, thien-2yl-CH, PhCH), $4.18\left(\mathrm{~d},{ }^{3} \mathrm{~J}=6.4 \mathrm{~Hz}, 1 \mathrm{H}, \mathrm{CHCO}_{2} \mathrm{Et}\right), 4.14-4.01(\mathrm{~m}, 2 \mathrm{H}$, $\left.\mathrm{CH}_{3} \mathrm{CH}_{2}\right), 1.20\left(\mathrm{t},{ }^{3} \mathrm{~J}=7.2 \mathrm{~Hz}, 3 \mathrm{H}, \mathrm{CH}_{3} \mathrm{CH}_{2}\right)$.

${ }^{13} \mathrm{C}$ NMR (101 MHz, $\mathrm{CDCl}_{3}$, C,F-decoupled): $\delta=199.2,195.3,170.1$, 142.4, 141.3, 136.6, 135.8, 133.7, 132.7, 131.6, 128.4, 127.9, 126.8, 124.6, 123.5, 123.2, 84.1, 78.2, 62.5, 58.8, 44.2, 42.8, 42.3, 14.0.

${ }^{19} \mathrm{~F}$ NMR $\left(564 \mathrm{MHz}, \mathrm{CDCl}_{3}\right): \delta=-71.9(\mathrm{~s}, 3 \mathrm{~F})$.

MS (EI, $70 \mathrm{eV}): m / z=527\left(6,\left[\mathrm{M}-\mathrm{NO}_{2}\right]^{+}\right), 481\left(8,\left[\mathrm{M}-\mathrm{NO}_{2}-\mathrm{EtOH}\right]^{+}\right)$, $463\left(9,\left[\mathrm{M}-\mathrm{NO}_{2}-\mathrm{EtOH}-\mathrm{H}_{2} \mathrm{O}\right]^{+}\right), 437\left(8,\left[\mathrm{M}-\mathrm{NO}_{2}-\mathrm{CO}_{2} \mathrm{Et}-\mathrm{H}_{2} \mathrm{O}\right]^{+}\right)$, 285 (47), 239 (100), 233 (69), 211 (13), 205 (8), 195 (17), 178 (25), 173 (26), 149 (25), $137(21), 115(20), 104(32), 91\left(20,\left[\mathrm{C}_{7} \mathrm{H}_{7}\right]^{+}\right), 77$ $\left(16,\left[\mathrm{C}_{6} \mathrm{H}_{5}\right]^{+}\right), 76\left(28,\left[\mathrm{C}_{6} \mathrm{H}_{4}\right]^{+}\right), 69\left(13,\left[\mathrm{CF}_{3}\right]^{+}\right)$.

MS (CI, methane): $m / z=602\left(1,[\mathrm{M}+\mathrm{Et}]^{+}\right), 574\left(11,[\mathrm{M}+\mathrm{H}]^{+}\right)$.

HRMS (ESI): $m / z[\mathrm{M}+\mathrm{Na}]^{+}$calcd for $\mathrm{C}_{28} \mathrm{H}_{22} \mathrm{~F}_{3} \mathrm{NO}_{7} \mathrm{SNa}^{+}: 596.0961$; found: 596.0960 .

tert-Butyl 3-\{(2S,3S,4S,5R,6R)-3-(Ethoxycarbonyl)-2-hydroxy-5-nitro-1',3'-dioxo-6-phenyl-2-(trifluoromethyl)-1',3'-dihydrospiro[cyclohexane-1,2'-indene]-4-yl\}-1H-indole-1-carboxylate (5g)

Yield: $190 \mathrm{mg}(54 \%, \mathrm{dr}=1.5: 1)$; yellowish solid; $28 \%$ ee [SFC: $(R, R)$ Whelk-01]; $R_{f}=0.24$ (n-pentane-Et $\left.{ }_{2} \mathrm{O}, 2: 1\right) ;[\alpha]_{\mathrm{D}}{ }^{22}+22.7(c=1.00, \mathrm{CH}-$ $\mathrm{Cl}_{3}$ ).

IR (ATR): 3359, 2976, 2295, 2079, 1719, 1558, 1453, 1366, 1249, 1159 , $849,753 \mathrm{~cm}^{-1}$.

${ }^{1} \mathrm{H} \mathrm{NMR}\left(600 \mathrm{MHz}, \mathrm{CDCl}_{3}\right): \delta=8.33(\mathrm{~s}, 1 \mathrm{H}, \mathrm{ArH}), 8.06\left(\mathrm{~d},{ }^{3} \mathrm{~J}=6.9 \mathrm{~Hz}\right.$, $1 \mathrm{H}, \mathrm{ArH}), 7.84\left(\mathrm{~d},{ }^{3} \mathrm{~J}=7.4 \mathrm{~Hz}, 1 \mathrm{H}, \mathrm{ArH}\right), 7.74-7.59$ (m, $\left.3 \mathrm{H}, \mathrm{ArH}\right), 7.42$ (d, $\left.{ }^{3}=7.9 \mathrm{~Hz}, 1 \mathrm{H}, \mathrm{ArH}\right), 7.30-7.18(\mathrm{~m}, 3 \mathrm{H}, \mathrm{ArH}), 7.08-6.87(\mathrm{~m}, 4 \mathrm{H}$, $\mathrm{ArH}$ ), 6.50 (dd, ${ }^{3} \mathrm{~J}=12.6 \mathrm{~Hz},{ }^{3} \mathrm{~J}=6.7 \mathrm{~Hz}, 1 \mathrm{H}, \mathrm{CHNO}_{2}$ ), 5.09 ('t', ${ }^{3} \mathrm{~J}=6.7$ $\mathrm{Hz}, 1 \mathrm{H}$, BocIndolyl-CH), $5.87(\mathrm{~s}, 1 \mathrm{H}, \mathrm{OH}), 4.87\left(\mathrm{~d},{ }^{3} \mathrm{~J}=12.6 \mathrm{~Hz}, 1 \mathrm{H}\right.$, $\mathrm{PhCH}), 4.28\left(\mathrm{~d},{ }^{3} \mathrm{~J}=6.7 \mathrm{~Hz}, 1 \mathrm{H}, \mathrm{CHCO}_{2} \mathrm{Et}\right), 3.90-3.77\left(\mathrm{~m}, 1 \mathrm{H}, \mathrm{CH}_{3} \mathrm{CH}_{2}\right)$, 3.70-3.59 (m, $\left.1 \mathrm{H}, \mathrm{CH}_{3} \mathrm{CH}_{2}\right), 1.74\left[\mathrm{~s}, 9 \mathrm{H}, \mathrm{C}\left(\mathrm{CH}_{3}\right)_{3}\right], 1.05\left(\mathrm{t},{ }^{3} \mathrm{~J}=7.2 \mathrm{~Hz}\right.$, $3 \mathrm{H}, \mathrm{CH}_{3} \mathrm{CH}_{2}$ ).
${ }^{13} \mathrm{C}$ NMR (101 MHz, $\mathrm{CDCl}_{3}$, C,F-decoupled): $\delta=199.3,195.2,170.4$, 149.6, 142.5, 141.3, 136.5, 135.8, 134.3, 133.0, 131.5, 128.6, 128.4, 127.7, 124.7, 124.6, 123.4, 123.2, 122.7, 117.3, 115.5, 111.2, 84.5, 83.3, 78.6, 62.4, 58.9, 43.4, 43.2, 35.7, 28.4, 13.7 .

${ }^{19} \mathrm{~F}$ NMR (376 MHz, $\left.\mathrm{CDCl}_{3}\right): \delta=-71.9(\mathrm{~s}, 3 \mathrm{~F})$.

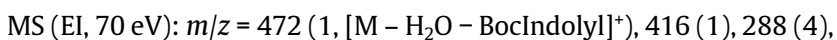
233 (42), 188 (3), 178 (3), 176 (5), 139 (5), 115 (10), 104 (8), 102 (5), $91\left(1,\left[\mathrm{C}_{7} \mathrm{H}_{7}\right]^{+}\right), 77\left(3,\left[\mathrm{C}_{6} \mathrm{H}_{5}\right]^{+}\right), 76\left(14,\left[\mathrm{C}_{6} \mathrm{H}_{4}\right]^{+}\right), 69\left(11,\left[\mathrm{CF}_{3}\right]^{+}\right), 57(100$, $\left.\left[\mathrm{C}_{4} \mathrm{H}_{9}\right]^{+}\right), 50(5)$.

MS (CI, methane): $m / z=707\left(4,[\mathrm{M}+\mathrm{H}]^{+}\right), 651\left(4,\left[\mathrm{M}-\mathrm{C}_{4} \mathrm{H}_{8}+\mathrm{H}\right]^{+}\right)$. HRMS (ESI): $m / z[\mathrm{M}+\mathrm{Na}]^{+}$calcd for $\mathrm{C}_{37} \mathrm{H}_{33} \mathrm{~F}_{3} \mathrm{~N}_{2} \mathrm{O}_{9} \mathrm{Na}^{+}:$729.2030; found: 729.2030 .

Ethyl $(2 R, 3 R, 4 S, 5 R, 6 R)-2-H y d r o x y-4-(n a p h t h a l e n-1-y l)-5-n i t r o-$ 1',3'-dioxo-6-phenyl-2-(trifluoromethyl)-1',3'-dihydrospiro[cyclohexane-1,2'-indene]-3-carboxylate ( $5 \mathrm{~h})$

Yield: $148 \mathrm{mg}(47 \%, \mathrm{dr}=20: 1)$; yellowish solid; $\mathrm{mp}>180{ }^{\circ} \mathrm{C} ; 89 \%$ ee [HPLC: Chiralpak IC]; $R_{f}=0.24$ (n-pentane-Et $\left.{ }_{2} \mathrm{O}, 2: 1\right) ;[\alpha]_{\mathrm{D}}^{22}-34.1(c=$ $\left.1.01, \mathrm{CHCl}_{3}\right)$.

IR (ATR): 3308, 2988, 2296, 2067, 1889, 1704, 1560, 1348, 1193, 1101 , $1018,861,770,698 \mathrm{~cm}^{-1}$.

${ }^{1} \mathrm{H}$ NMR $\left(600 \mathrm{MHz}, \mathrm{CDCl}_{3}\right): \delta=8.74\left(\mathrm{~d},{ }^{3} \mathrm{~J}=8.4 \mathrm{~Hz}, 1 \mathrm{H}, \mathrm{ArH}\right), 8.12(\mathrm{~d}$, $3 J=7.9 \mathrm{~Hz}, 1 \mathrm{H}, \mathrm{ArH}), 7.73-7.91(\mathrm{~m}, 6 \mathrm{H}, \mathrm{ArH}), 7.61-7.55(\mathrm{~m}, 1 \mathrm{H}$, ArH), $7.46\left(\mathrm{~d},{ }^{3} \mathrm{~J}=7.4 \mathrm{~Hz}, 1 \mathrm{H}, \mathrm{ArH}\right), 7.41-7.35(\mathrm{~m}, 1 \mathrm{H}, \mathrm{ArH}), 7.15-7.10$ $(\mathrm{m}, 2 \mathrm{H}, \mathrm{ArH}), 7.08-7.01(\mathrm{~m}, 3 \mathrm{H}, \mathrm{ArH}), 6.11(\mathrm{~s}, 1 \mathrm{H}, \mathrm{OH}), 6.00(\mathrm{dd}$, $\left.{ }^{3} \mathrm{~J}=10.9 \mathrm{~Hz},{ }^{3} \mathrm{~J}=7.7 \mathrm{~Hz}, 1 \mathrm{H}, \mathrm{CHNO}_{2}\right), 5.81\left(\mathrm{dd},{ }^{3} \mathrm{~J}=12.6 \mathrm{~Hz},{ }^{3} \mathrm{~J}=7.7 \mathrm{~Hz}\right.$, $1 \mathrm{H}$, naphthyl-CH), 4.56-4.48 (m, $\left.2 \mathrm{H}, \mathrm{PhCH}, \mathrm{CHCO}_{2} \mathrm{Et}\right), 4.01-3.81$ (m, $\left.2 \mathrm{H}, \mathrm{CH}_{3} \mathrm{CH}_{2}\right), 1.09\left(\mathrm{t},{ }^{3} \mathrm{~J}=7.2 \mathrm{~Hz}, 3 \mathrm{H}, \mathrm{CH}_{3} \mathrm{CH}_{2}\right)$.

${ }^{13} \mathrm{C}$ NMR (101 MHz, $\mathrm{CDCl}_{3}$, C,F-decoupled): $\delta=198.2,195.2,173.4$, 143.7, 143.3, 136.3, 136.2, 134.2, 133.9, 132.4, 130.1, 129.7, 129.4, 129.0, 128.7, 127.9, 126.3, 125.7, 124.8, 123.7, 123.6, 122.6, 92.4, 79.6, $63.4,62.7,46.3,43.0,36.7,13.7$.

$\left.{ }^{19} \mathrm{~F} \mathrm{NMR} \mathrm{(376} \mathrm{MHz,} \mathrm{CDCl}_{3}\right): \delta=-73.9(\mathrm{~s}, 3 \mathrm{~F})$.

MS (EI, $70 \mathrm{eV}): m / z=617\left(8,[\mathrm{M}]^{+}\right), 571\left(2,\left[\mathrm{M}-\mathrm{NO}_{2}\right]^{+}\right), 507(20,[\mathrm{M}-$ $\left.\left.\mathrm{NO}_{2}-\mathrm{EtOH}-\mathrm{H}_{2} \mathrm{O}\right]^{+}\right), 383\left(25,\left[\mathrm{M}-\mathrm{NO}_{2}-\mathrm{EtOH}-\mathrm{OH}-\mathrm{Naph}\right]^{+}\right), 283$ (9), 233 (100), 176 (5), $152(31), 115(6), 104(10), 91\left(1,\left[\mathrm{C}_{7} \mathrm{H}_{7}\right]^{+}\right), 87$ (14), $77\left(9,\left[\mathrm{C}_{6} \mathrm{H}_{5}\right]^{+}\right), 76\left(50,\left[\mathrm{C}_{6} \mathrm{H}_{4}\right]^{+}\right), 69\left(24,\left[\mathrm{CF}_{3}\right]^{+}\right), 50(9)$.

MS (CI, methane): $m / z=646\left(3,[\mathrm{M}+\mathrm{Et}]^{+}\right), 618\left(40,[\mathrm{M}+\mathrm{H}]^{+}\right)$.

HRMS (ESI): $m / z[\mathrm{M}+\mathrm{Na}]^{+}$calcd for $\mathrm{C}_{34} \mathrm{H}_{26} \mathrm{~F}_{3} \mathrm{NO}_{7} \mathrm{Na}^{+}: 640.1554$; found: 640.1552 .

Ethyl (2S,3S,4S,5R,6R)-6-(4-Bromophenyl)-2-hydroxy-5-nitro1',3'-dioxo-4-phenyl-2-(trifluoromethyl)-1',3'-dihydrospiro[cyclohexane-1,2'-indene]-3-carboxylate ( $5 \mathrm{i})$

Yield: $233 \mathrm{mg}(72 \%, \mathrm{dr}=1.3: 1)$; colorless solid; 0\% ee [SFC: $(R, R)$ Whelk-01]; $R_{f}=0.17$ (n-pentane-Et ${ }_{2} \mathrm{O}, 2: 1$ ).

IR (ATR): 3355, 2984, 2662, 2330, 2102, 1923, 1708, 1557, 1466, 1368 , $1253,1179,1020,886,757,701 \mathrm{~cm}^{-1}$.

${ }^{1} \mathrm{H}$ NMR $\left(600 \mathrm{MHz}, \mathrm{CDCl}_{3}\right): \delta$ (mixture of diastereomers $)=7.97(\mathrm{~d}$, $3 J=7.9 \mathrm{~Hz}, 1 \mathrm{H}, \mathrm{ArH}), 7.93-7.86(\mathrm{~m}, 2 \mathrm{H}, \mathrm{ArH}), 7.84-7.64(\mathrm{~m}, 6 \mathrm{H}$, ArH), 7.51-7.28 (m, $11 \mathrm{H}$, ArH), $7.12\left(\mathrm{dd},{ }^{3} J=7.9 \mathrm{~Hz},{ }^{3} J=1.5 \mathrm{~Hz}, 1 \mathrm{H}\right.$, ArH), 7.05-6.99 (m, $1 \mathrm{H}, \mathrm{ArH}), 6.95-6.86(\mathrm{~m}, 2 \mathrm{H}, \mathrm{ArH}), 6.85-6.69(\mathrm{~m}$, $2 \mathrm{H}, \mathrm{ArH}), 6.34^{\mathrm{a}}\left(\mathrm{dd},{ }^{3} \mathrm{~J}=12.9 \mathrm{~Hz},{ }^{3} \mathrm{~J}=7.4 \mathrm{~Hz}, 1 \mathrm{H}, \mathrm{CHNO}_{2}\right), 6.27(\mathrm{~s}, 1 \mathrm{H}$, $\mathrm{OH}), 6.16^{\mathrm{a}}(\mathrm{s}, 1 \mathrm{H}, \mathrm{OH}), 6.01\left(\mathrm{dd},{ }^{3} \mathrm{~J}=11.6 \mathrm{~Hz},{ }^{3} \mathrm{~J}=8.2 \mathrm{~Hz}, 1 \mathrm{H}, \mathrm{CHNO}_{2}\right.$ ), $5.88^{\mathrm{a}}\left(\mathrm{d},{ }^{3} \mathrm{~J}=12.9 \mathrm{~Hz}, 1 \mathrm{H}, 4-\mathrm{BrC}_{6} \mathrm{H}_{4} \mathrm{CH}\right), 5.28\left(\mathrm{~d},{ }^{3} \mathrm{~J}=11.6 \mathrm{~Hz}, 1 \mathrm{H}, 4-\right.$ $\mathrm{BrC}_{6} \mathrm{H}_{4} \mathrm{CH}$ ), 4.86 (dd, ${ }^{3} \mathrm{~J}=12.1 \mathrm{~Hz},{ }^{3} \mathrm{~J}=8.2 \mathrm{~Hz}, 1 \mathrm{H}, \mathrm{PhCH}$ ), $4.58^{\mathrm{a}}$ ('t', 
$\left.{ }^{3} J=7.4 \mathrm{~Hz}, 1 \mathrm{H}, \mathrm{PhCH}\right), 4.43\left(\mathrm{~d},{ }^{3} \mathrm{~J}=12.1 \mathrm{~Hz}, 1 \mathrm{H}, \mathrm{CHCO}_{2} \mathrm{Et}\right), 4.25^{\mathrm{a}}(\mathrm{d}$, $\left.3 \mathrm{~J}=7.4 \mathrm{~Hz}, 1 \mathrm{H}, \mathrm{CHCO}_{2} \mathrm{Et}\right), 4.02-3.84\left(\mathrm{~m}, 4 \mathrm{H}, \mathrm{CH}_{3} \mathrm{CH}_{2}\right), 1.13\left(\mathrm{t},{ }^{3} \mathrm{~J}=7.2\right.$ $\left.\mathrm{Hz}, 3 \mathrm{H}, \mathrm{CH}_{3} \mathrm{CH}_{2}\right), 1.05\left(\mathrm{t},{ }^{3} \mathrm{~J}=7.2 \mathrm{~Hz}, 3 \mathrm{H}, \mathrm{CH}_{3} \mathrm{CH}_{2}\right)$.

${ }^{13} \mathrm{C}$ NMR (101 MHz, $\mathrm{CDCl}_{3}$, C,F-decoupled): $\delta$ (mixture of diastereomers $)=199.9,199.1,195.9,192.8,172.3,170.6,143.1,142.9,142.6$, 141.7, 136.8, 136.5, 136.4, 135.9, 134.6, 134.2, 134.1, 133.8, 133.2, 133.0, 131.3, 129.8, 129.7, 129.5, 129.3, 129.1, 128.9, 128.8, 128.5, $128.1,127.2,126.7,124.0,123.8,123.4,123.3,90.7,85.1^{\text {a }}, 79.6,78.6^{a}$, $62.5,62.4,61.6,58.4,47.1^{\mathrm{a}}, 44.3,44.2^{\mathrm{a}}, 43.8,43.2,40.8^{\mathrm{a}}$, 13.9, 13.6 .

$\left.{ }^{19} \mathrm{~F} \mathrm{NMR} \mathrm{(376} \mathrm{MHz}, \mathrm{CDCl}_{3}\right): \delta$ (mixture of diastereomers) $=-71.9(\mathrm{~s}, 3$ F), $-73.5(\mathrm{~s}, 3 \mathrm{~F})$.

MS (EI, $70 \mathrm{eV}): m / z=599\left(14,\left[\mathrm{M}-\mathrm{NO}_{2}\right]^{+}\right), 553\left(8,\left[\mathrm{M}-\mathrm{NO}_{2}-\mathrm{EtOH}\right]^{+}\right)$, 535 (6, [ $\left.\left.\mathrm{M}-\mathrm{NO}_{2}-\mathrm{EtOH}-\mathrm{H}_{2} \mathrm{O}\right]^{+}\right), 507$ (4), 428 (7), 359 (5), 313 (7), 249 (6), 233 (100), 189 (20), 176 (10), 161 (5), 115 (6), 104 (7), 91 (4, $\left.\left[\mathrm{C}_{7} \mathrm{H}_{7}\right]^{+}\right), 77\left(5,\left[\mathrm{C}_{6} \mathrm{H}_{5}\right]^{+}\right), 76\left(6,\left[\mathrm{C}_{6} \mathrm{H}_{4}\right]^{+}\right), 69\left(2,\left[\mathrm{CF}_{3}\right]^{+}\right)$.

MS (CI, methane): $m / z=646\left(2,[\mathrm{M}+\mathrm{H}]^{+}\right)$.

HRMS (ESI): $m / z[\mathrm{M}+\mathrm{Na}]^{+}$calcd for $\mathrm{C}_{30} \mathrm{H}_{23}{ }^{79} \mathrm{BrF}_{3} \mathrm{NO}_{7} \mathrm{Na}^{+}: 668.0502$; found: 668.0515 .

\section{Ethyl (2S,3S,4S,5R,6R)-2-Hydroxy-5-nitro-6-(4-nitrophenyl)-1',3'- dioxo-4-phenyl-2-(trifluoromethyl)-1',3'-dihydrospiro[cyclohex- ane-1,2'-indene]-3-carboxylate (5j)}

Yield: $70 \mathrm{mg}$ ( $23 \%, \mathrm{dr}=1.5: 1)$; yellow solid; $81 \%$ ee [SFC: $(R, R)$-Whelk01 ]; $R_{f}=0.21$ (n-pentane- $\mathrm{Et}_{2} \mathrm{O}, 2: 1$ ).

IR (ATR): 3761, 3399, 3098, 2689, 2440, 2292, 2209, 2091, 2000, 1912, $1691,1596,1512,1423,1340,1247,1184,997,855,736 \mathrm{~cm}^{-1}$.

${ }^{1} \mathrm{H}$ NMR $\left(600 \mathrm{MHz}, \mathrm{CDCl}_{3}\right): \delta=8.55\left(\mathrm{~d},{ }^{3} J=8.9 \mathrm{~Hz}, 1 \mathrm{H}, \mathrm{ArH}\right), 8.38-$ $8.31(\mathrm{~m}, 1 \mathrm{H}, \mathrm{ArH}), 8.07\left(\mathrm{dd},{ }^{3} \mathrm{~J}=5.9 \mathrm{~Hz},{ }^{4} \mathrm{~J}=3.0 \mathrm{~Hz}, 1 \mathrm{H}, \mathrm{ArH}\right), 7.92-$ 7.86 (m, 2 H, ArH), 7.82-7.70 (m, 3 H, ArH), 7.63 (br s, 1 H, ArH), 7.547.41 (m, $1 \mathrm{H}, \mathrm{ArH}), 7.36-7.31$ (m, $2 \mathrm{H}, \mathrm{ArH}), 7.09$ (br s, $1 \mathrm{H}, \mathrm{ArH}), 6.49$ (dd, $\left.{ }^{3} \mathrm{~J}=12.9 \mathrm{~Hz},{ }^{3} \mathrm{~J}=7.7 \mathrm{~Hz}, 1 \mathrm{H}, \mathrm{CHNO}_{2}\right), 6.14(\mathrm{~s}, 1 \mathrm{H}, \mathrm{OH}), 5.15(\mathrm{~d}$, ${ }^{3} J=12.9 \mathrm{~Hz}, 1 \mathrm{H}, 4-\mathrm{NO}_{2} \mathrm{C}_{6} \mathrm{H}_{4} \mathrm{CH}$ ), 4.61 ('t', ${ }^{3} \mathrm{~J}=7.7 \mathrm{~Hz}, 1 \mathrm{H}, \mathrm{PhCH}$ ), 4.16 $\left(\mathrm{d},{ }^{3} \mathrm{~J}=7.7 \mathrm{~Hz}, 1 \mathrm{H}, \mathrm{CHCO}_{2} \mathrm{Et}\right), 4.02-3.88\left(\mathrm{~m}, 2 \mathrm{H}, \mathrm{CH}_{3} \mathrm{CH}_{2}\right), 1.16(\mathrm{t}$, $\left.3 \mathrm{~J}=7.2 \mathrm{~Hz}, 3 \mathrm{H}, \mathrm{CH}_{3} \mathrm{CH}_{2}\right)$.

${ }^{13} \mathrm{C}$ NMR (101 MHz, $\mathrm{CDCl}_{3}$, C,F-decoupled): $\delta=198.9,194.1,170.7$, 147.6, 142.8, 140.9, 140.4, 138.6, 137.4, 136.4, 136.2, 136.1, 134.4, $132.9,132.4,131.1,129.5,128.9,123.9,123.9,123.8,123.7,123.5$, 84.0, 78.2, 62.7, 58.8, 46.5, 43.9, 42.7, 13.9 .

$\left.{ }^{19} \mathrm{~F} \mathrm{NMR} \mathrm{(376} \mathrm{MHz,} \mathrm{CDCl}_{3}\right): \delta=-72.1(\mathrm{~s}, 3 \mathrm{~F})$.

MS (EI, $70 \mathrm{eV}): m / z=566\left(7,\left[\mathrm{M}-\mathrm{NO}_{2}\right]^{+}\right), 520\left(2,\left[\mathrm{M}-\mathrm{NO}_{2}-\mathrm{EtOH}\right]^{+}\right)$, $502\left(3,\left[\mathrm{M}-\mathrm{NO}_{2}-\mathrm{EtOH}-\mathrm{H}_{2} \mathrm{O}\right]^{+}\right), 474\left(2,\left[\mathrm{M}-\mathrm{NO}_{2}-\mathrm{CO}_{2}-\mathrm{EtOH}\right]^{+}\right)$, 279 (97), 262 (75), 232 (100), 189 (39), 176 (56), 149 (15), 115 (37), $104(51), 91\left(44,\left[\mathrm{C}_{7} \mathrm{H}_{7}\right]^{+}\right), 77\left(69,\left[\mathrm{C}_{6} \mathrm{H}_{5}\right]^{+}\right), 76\left(64,\left[\mathrm{C}_{6} \mathrm{H}_{4}\right]^{+}\right), 69(56$, $\left.\left[\mathrm{CF}_{3}\right]^{+}\right), 51(44)$.

MS (CI, methane): $m / z=641\left(1,[\mathrm{M}+\mathrm{Et}]^{+}\right), 613\left(22,[\mathrm{M}+\mathrm{H}]^{+}\right)$.

HRMS (ESI): $m / z[\mathrm{M}+\mathrm{Na}]^{+}$calcd for $\mathrm{C}_{30} \mathrm{H}_{23} \mathrm{~F}_{3} \mathrm{~N}_{2} \mathrm{O}_{9} \mathrm{Na}^{+}$: 635.1248; found: 635.1249 .

Ethyl (2R,3R,4S,5R,6R)-2-Hydroxy-6-(3-methoxyphenyl)-5-nitro1',3'-dioxo-4-phenyl-2-(trifluoromethyl)-1',3'-dihydrospiro[cyclohexane-1,2'-indene]-3-carboxylate (5k)

Yield: $141 \mathrm{mg}(47 \%, \mathrm{dr}=1.4: 1)$; colorless solid; $\mathrm{mp} 179-181^{\circ} \mathrm{C} ; 92 \%$ ee [HPLC: Chiralpak AD]; $R_{f}=0.19$ (n-pentane-Et $\left.{ }_{2} \mathrm{O}, 2: 1\right)$; $[\alpha]_{\mathrm{D}}{ }^{22}-37.1$ ( $c=0.46, \mathrm{CHCl}_{3}$ ).

IR (ATR): 3409, 2979, 2658, 2328, 2070, 1714, 1560, 1461, 1371, 1173 , $1021,759,700 \mathrm{~cm}^{-1}$.
${ }^{1} \mathrm{H}$ NMR $\left(600 \mathrm{MHz}, \mathrm{CDCl}_{3}\right): \delta=7.98\left(\mathrm{~d},{ }^{3} \mathrm{~J}=7.4 \mathrm{~Hz}, 1 \mathrm{H}, \mathrm{ArH}\right), 7.89-$ 7.73 (m, 3 H, ArH), 7.43-7.37 (m, 2 H, ArH), 7.36-7.28 (m, 3 H, ArH), 7.01-6.88 (m, $1 \mathrm{H}, \mathrm{ArH}), 6.63\left(\mathrm{~d},{ }^{3} \mathrm{~J}=7.9 \mathrm{~Hz}, 1 \mathrm{H}, \mathrm{ArH}\right), 6.60-6.54(\mathrm{~m}, 2$ $\mathrm{H}, \mathrm{ArH}), 6.19(\mathrm{~s}, 1 \mathrm{H}, \mathrm{OH}), 5.92\left(\mathrm{dd},{ }^{3} \mathrm{~J}=10.9 \mathrm{~Hz},{ }^{3} \mathrm{~J}=8.2 \mathrm{~Hz}, 1 \mathrm{H}, \mathrm{CH}-\right.$ $\mathrm{NO}_{2}$ ), 4.83 (dd, $\left.{ }^{3} \mathrm{~J}=12.6 \mathrm{~Hz},{ }^{3} \mathrm{~J}=8.2 \mathrm{~Hz}, 1 \mathrm{H}, \mathrm{PhCH}\right), 4.43$ (d, ${ }^{3} \mathrm{~J}=10.9$ $\mathrm{Hz}, 1 \mathrm{H}, 3-\mathrm{MeOC}_{6} \mathrm{H}_{4} \mathrm{CH}$ ), 4.27 (d, $\left.{ }^{3} \mathrm{~J}=12.6 \mathrm{~Hz}, 1 \mathrm{H}, \mathrm{CHCO}_{2} \mathrm{Et}\right), 4.00-3.89$ (m, $1 \mathrm{H}, \mathrm{CH}_{3} \mathrm{CH}_{2}$ ), 3.88-3.77 (m, $\left.1 \mathrm{H}, \mathrm{CH}_{3} \mathrm{CH}_{2}\right), 3.61\left(\mathrm{~s}, 3 \mathrm{H}, \mathrm{OCH}_{3}\right), 1.03$ (t, ${ }^{3} \mathrm{~J}=7.2 \mathrm{~Hz}, 3 \mathrm{H}, \mathrm{CH}_{3} \mathrm{CH}_{2}$ ).

${ }^{13} \mathrm{C}$ NMR (101 MHz, $\mathrm{CDCl}_{3}$, C-F-decoupled): $\delta=198.0,195.3,172.7$, $159.4,143.1,142.9,136.1,136.0,135.2,133.5,129.7,128.9,128.6$, 124.6, 123.5, 123.3, 114.3, 91.6, 79.1, 62.4, 62.2, 55.1, 46.3, 43.1, 42.4, 13.4.

${ }^{19} \mathrm{~F} \mathrm{NMR}\left(376 \mathrm{MHz}, \mathrm{CDCl}_{3}\right): \delta=-72.0(\mathrm{~s}, 3 \mathrm{~F})$.

MS (EI, $70 \mathrm{eV}): m / z=597\left(2,[\mathrm{M}]^{++}\right), 551\left(16,\left[\mathrm{M}-\mathrm{NO}_{2}\right]^{+}\right), 533(3,[\mathrm{M}-$ $\left.\left.\mathrm{NO}_{2}-\mathrm{H}_{2} \mathrm{O}\right]^{+}\right), 505\left(18,\left[\mathrm{M}-\mathrm{NO}_{2}-\mathrm{EtOH}\right]^{+}\right), 487\left(14,\left[\mathrm{M}-\mathrm{NO}_{2}-\mathrm{EtOH}-\right.\right.$ $\left.\left.\mathrm{H}_{2} \mathrm{O}\right]^{+}\right), 459\left(44,\left[\mathrm{M}-\mathrm{NO}_{2}-\mathrm{OMe}-\mathrm{EtO}-\mathrm{OH}\right]^{+}\right), 439$ (7), 395 (7), 391 (7), 309 (22), 263 (100), 243 (13), 237 (9), 235 (21), 233 (20), 221 (31), 205 (7), 202 (11), 189 (22), 178 (14), 173 (36), 165 (32), 161 (8), 159 (11), 155 (7), 151 (7), 149 (21), 145 (11), 135 (8), 133 (16), 131 (13), 121 (26), 115 (30), $104(31), 91\left(40,\left[\mathrm{C}_{7} \mathrm{H}_{7}\right]^{+}\right), 89(8), 77\left(27,\left[\mathrm{C}_{6} \mathrm{H}_{5}\right]^{+}\right)$, $76\left(16,\left[\mathrm{C}_{6} \mathrm{H}_{4}\right]^{+}\right), 69\left(10,\left[\mathrm{CF}_{3}\right]^{+}\right)$.

MS (CI, methane): $m / z=626\left(1,[\mathrm{M}+\mathrm{Et}]^{+}\right), 598\left(6,[\mathrm{M}+\mathrm{H}]^{+}\right)$.

HRMS (ESI): $m / z[\mathrm{M}+\mathrm{Na}]^{+}$calcd for $\mathrm{C}_{31} \mathrm{H}_{26} \mathrm{~F}_{3} \mathrm{NO}_{8} \mathrm{Na}^{+}$: 620.1503; found: 620.1508 .

Ethyl (2S,3S,4S,5R,6R)-2-Hydroxy-5-nitro-1',3'-dioxo-4-phenyl-6( $p$-tolyl)-2-(trifluoromethyl)-1',3'-dihydrospiro[cyclohexane-1,2'indene]-3-carboxylate (5l)

Yield: $101 \mathrm{mg}$ (35\%, dr = 1:1); colorless solid; $\mathrm{mp} 140{ }^{\circ} \mathrm{C}$ (dec.); $75 \%$ ee [HPLC: Chiralpak IC]; $R_{f}=0.20$ (n-pentane-Et $\left.{ }_{2} \mathrm{O}, 2: 1\right) ;[\alpha]_{\mathrm{D}}^{22}-34.5(c=$ $\left.0.20, \mathrm{CHCl}_{3}\right)$.

IR (ATR): 3363, 2984, 2656, 2324, 2070, 1976, 1709, 1554, 1458, 1354, $1186,1022,890,756,704 \mathrm{~cm}^{-1}$.

${ }^{1} \mathrm{H}$ NMR $\left(600 \mathrm{MHz}, \mathrm{CDCl}_{3}\right): \delta$ (mixture of diastereomers) $=7.97(\mathrm{~d}$, $\left.{ }^{3} J=7.4 \mathrm{~Hz}, 1 \mathrm{H}, \mathrm{ArH}\right), 7.90-7.53(\mathrm{~m}, 9 \mathrm{H}, \mathrm{ArH}), 7.46-7.28(\mathrm{~m}, 8 \mathrm{H}$, ArH), 7.06 (br s, $1 \mathrm{H}, \mathrm{ArH}), 6.99-6.55(\mathrm{~m}, 7 \mathrm{H}, \mathrm{ArH}), 6.43^{\mathrm{a}}\left(\mathrm{dd},{ }^{3} \mathrm{~J}=12.9\right.$ $\left.\mathrm{Hz},{ }^{3} \mathrm{~J}=7.7 \mathrm{~Hz}, 1 \mathrm{H}, \mathrm{CHNO}_{2}\right), 6.18(\mathrm{~s}, 1 \mathrm{H}, \mathrm{OH}), 6.03^{\mathrm{a}}(\mathrm{s}, 1 \mathrm{H}, \mathrm{OH}), 5.92$ $\left(\mathrm{dd},{ }^{3} \mathrm{~J}=11.1 \mathrm{~Hz},{ }^{3} \mathrm{~J}=8.2 \mathrm{~Hz}, 1 \mathrm{H}, \mathrm{CHNO}_{2}\right), 4.97^{\mathrm{a}}\left(\mathrm{d},{ }^{3} \mathrm{~J}=12.9 \mathrm{~Hz}, 1 \mathrm{H}, p-\right.$ TolCH), $4.82\left(\mathrm{dd},{ }^{3} \mathrm{~J}=12.6 \mathrm{~Hz},{ }^{3} \mathrm{~J}=8.2 \mathrm{~Hz}, 1 \mathrm{H}, \mathrm{PhCH}\right), 4.56^{\mathrm{a}}$ ('t', ${ }^{3} \mathrm{~J}=7.7$ $\mathrm{Hz}, 1 \mathrm{H}, \mathrm{PhCH}), 4.42$ (d, $\left.{ }^{3} \mathrm{~J}=11.1 \mathrm{~Hz}, 1 \mathrm{H}, p-\mathrm{TolCH}\right), 4.27$ (d, ${ }^{3} \mathrm{~J}=12.9 \mathrm{~Hz}$, $\left.1 \mathrm{H}, \mathrm{CHCO}_{2} \mathrm{Et}\right), 4.20^{\mathrm{a}}\left(\mathrm{d},{ }^{3} \mathrm{~J}=7.7 \mathrm{~Hz}, 1 \mathrm{H}, \mathrm{CHCO}_{2} \mathrm{Et}\right), 3.99-3.76(\mathrm{~m}, 4 \mathrm{H}$, $\left.\mathrm{CH}_{3} \mathrm{CH}_{2}\right), 2.10\left(\mathrm{~s}, 3 \mathrm{H}, \mathrm{ArCH}_{3}\right), 2.01\left(\mathrm{~s}, 3 \mathrm{H}, \mathrm{ArCH}_{3}\right), 1.13\left(\mathrm{t},{ }^{3} \mathrm{~J}=7.2 \mathrm{~Hz}, 3\right.$ $\left.\mathrm{H}, \mathrm{CH}_{3} \mathrm{CH}_{2}\right), 1.02\left(\mathrm{t},{ }^{3} \mathrm{~J}=7.2 \mathrm{~Hz}, 3 \mathrm{H}, \mathrm{CH}_{3} \mathrm{CH}_{2}\right)$.

${ }^{13} \mathrm{C}$ NMR (101 MHz, $\mathrm{CDCl}_{3}, \mathrm{C}, \mathrm{F}-$ decoupled): $\delta$ (mixture of diastereomers $)=199.4,198.2,195.6,195.1,172.9,170.7,143.4,143.1142 .6$, 141.3, 138.4, 138.1, 136.5, 136.2, 136.1, 135.7, 133.7, 133.5, 131.3, 130.8, 129.8, 129.6, 129.4, 129.1, 129.0, 128.8, 128.6, 124.7, 123.7, $123.5,123.5,123.2,92.0,84.3^{a}, 79.3,78.2^{2}, 62.5,62.4,58.9,46.7^{\mathrm{a}}, 46.0$, 44.1 ${ }^{\mathrm{a}}, 43.2,42.6,21.0,20.9,13.9,13.5$.

${ }^{19} \mathrm{~F} \operatorname{NMR}\left(376 \mathrm{MHz}, \mathrm{CDCl}_{3}\right): \delta$ (mixture of diastereomers) $=-72.1(\mathrm{~s}, 3$ F), $-73.9(\mathrm{~s}, 3 \mathrm{~F})$.

MS (EI, $70 \mathrm{eV}): m / z=581\left(1,[\mathrm{M}]^{+}\right), 535\left(93,\left[\mathrm{M}-\mathrm{NO}_{2}\right]^{+}\right), 489(51,[\mathrm{M}-$ $\left.\left.\mathrm{NO}_{2}-\mathrm{EtOH}\right]^{+}\right), 471\left(60,\left[\mathrm{M}-\mathrm{NO}_{2}-\mathrm{EtOH}-\mathrm{H}_{2} \mathrm{O}\right]^{+}\right), 443(31), 423(9)$, 379 (34), 351 (10), 327 (12), 293 (36), 247 (100), 233 (47), 219 (12), 205 (15), 202 (9), 191 (10), 189 (29), 173 (14), 149 (12), 115 (22), 105 (20), $91\left(17,\left[\mathrm{C}_{7} \mathrm{H}_{7}\right]^{+}\right), 77\left(14,\left[\mathrm{C}_{6} \mathrm{H}_{5}\right]^{+}\right), 76\left(12,\left[\mathrm{C}_{6} \mathrm{H}_{4}\right]^{+}\right), 69\left(7,\left[\mathrm{CF}_{3}\right]^{+}\right)$.

MS (CI, methane): $m / z=582\left(2,[\mathrm{M}+\mathrm{H}]^{+}\right)$.

HRMS (ESI): $m / z[\mathrm{M}+\mathrm{Na}]^{+}$calcd for $\mathrm{C}_{31} \mathrm{H}_{26} \mathrm{~F}_{3} \mathrm{NO}_{7} \mathrm{Na}^{+}$: 604.1554; found: 604.1558 . 
Methyl (2R,3R,4S,5R,6R)-2-Hydroxy-5-nitro-1',3'-dioxo-4,6-diphenyl-2-(trifluoromethyl)-1',3'-dihydrospiro[cyclohexane-1,2'-indene]-3-carboxylate $(5 \mathrm{~m})$

Yield: $143 \mathrm{mg}(52 \%, \mathrm{dr}=1: 1)$; colorless solid; $86 \%$ ee [HPLC: Chiralpak $\mathrm{IC}$; $R_{f}=0.18$ (n-pentane-Et ${ }_{2} \mathrm{O}, 2: 1$ ).

IR (ATR): 3368, 3039, 2954, 2301, 2074, 1894, 1707, 1556, 1444, 1352 , $1251,1179,1031,842,759,700 \mathrm{~cm}^{-1}$.

${ }^{1} \mathrm{H}$ NMR $\left(600 \mathrm{MHz}, \mathrm{CDCl}_{3}\right): \delta$ (mixture of diastereomers) $=7.94(\mathrm{~d}$, $\left.{ }^{3} J=7.9 \mathrm{~Hz}, 1 \mathrm{H}, \mathrm{ArH}\right), 7.88-7.55(\mathrm{~m}, 10 \mathrm{H}, \mathrm{ArH}), 7.54-7.08(\mathrm{~m}, 13 \mathrm{H}$, ArH), 7.01-6.70 (m, $4 \mathrm{H}, \mathrm{ArH}), 6.44^{\mathrm{a}}\left(\mathrm{dd},{ }^{3} \mathrm{~J}=13.1 \mathrm{~Hz},{ }^{3} \mathrm{~J}=7.7 \mathrm{~Hz}, 1 \mathrm{H}\right.$, $\left.\mathrm{CHNO}_{2}\right), 6.06(\mathrm{~s}, 1 \mathrm{H}, \mathrm{OH}), 6.01^{\mathrm{a}}(\mathrm{s}, 1 \mathrm{H}, \mathrm{OH}), 5.98\left(\mathrm{dd},{ }^{3} \mathrm{~J}=11.4 \mathrm{~Hz}\right.$, $\left.{ }^{3} J=8.2 \mathrm{~Hz}, 1 \mathrm{H}, \mathrm{CHNO}_{2}\right), 5.01^{\mathrm{a}}\left(\mathrm{d},{ }^{3} J=13.1 \mathrm{~Hz}, 1 \mathrm{H}, \mathrm{PhCH}\right), 4.84(\mathrm{dd}$, ${ }^{3} J=12.6 \mathrm{~Hz},{ }^{3} \mathrm{~J}=8.2 \mathrm{~Hz}, 1 \mathrm{H}, \mathrm{PhCH}$ ), $4.58^{\mathrm{a}}$ ('t', ${ }^{3} \mathrm{~J}=7.7 \mathrm{~Hz}, 1 \mathrm{H}, \mathrm{PhCH}$ ), $4.46\left(\mathrm{~d},{ }^{3} \mathrm{~J}=11.4 \mathrm{~Hz}, 1 \mathrm{H}, \mathrm{PhCH}\right), 4.33\left(\mathrm{~d},{ }^{3} \mathrm{~J}=12.6 \mathrm{~Hz}, 1 \mathrm{H}, \mathrm{CHCO}_{2} \mathrm{Me}\right)$, $4.25^{\mathrm{a}}\left(\mathrm{d}, 3^{3}=7.7 \mathrm{~Hz}, 1 \mathrm{H}, \mathrm{CHCO}_{2} \mathrm{Me}\right), 3.53\left(\mathrm{~s}, 3 \mathrm{H}, \mathrm{OCH}_{3}\right), 3.45(\mathrm{~s}, 3 \mathrm{H}$, $\left.\mathrm{OCH}_{3}\right)$.

${ }^{13} \mathrm{C}$ NMR (101 MHz, $\mathrm{CDCl}_{3}$, C,F-decoupled): $\delta$ (mixture of diastereomers $)=199.3,198.4,195.6,195.1,173.1,171.3,143.2,143.0,142.6$, $141.3,136.6,136.3,136.3,135.8,133.8,133.0,131.1,129.5,129.2$, $129.1,128.9,128.7,128.6,128.5,124.7,124.7,123.7,123.5,123.5$, $123.2,91.3,84.1^{\mathrm{a}}, 79.3,78.3^{\mathrm{a}}, 62.1,58.9^{\mathrm{a}}, 53.0,52.9,46.7^{\mathrm{a}}, 46.6,44.1^{\mathrm{a}}$, 43.6, 43.0, 42.7.

$\left.{ }^{19} \mathrm{~F} \mathrm{NMR} \mathrm{(376} \mathrm{MHz,} \mathrm{CDCl}_{3}\right): \delta$ (mixture of diastereomers) $=-72.1(\mathrm{~s}, 3$ F), $-73.9(\mathrm{~s}, 3 \mathrm{~F})$.

MS (EI, $70 \mathrm{eV}): m / z=507\left(51,\left[\mathrm{M}-\mathrm{NO}_{2}\right]^{+}\right), 475\left(28,\left[\mathrm{M}-\mathrm{NO}_{2}-\right.\right.$ $\left.\mathrm{MeOH}]^{+}\right), 458\left(32,\left[\mathrm{M}-\mathrm{NO}_{2}-\mathrm{MeOH}-\mathrm{H}_{2} \mathrm{O}\right]^{+}\right), 429\left(17,\left[\mathrm{M}-\mathrm{NO}_{2}-\mathrm{CO}\right.\right.$ - $\left.\mathrm{MeOH}-\mathrm{H}_{2} \mathrm{O}\right]^{+}$), 365 (13), 265 (24), 233 (100), 205 (14), 191 (11), 178 (10), 173 (13), $149(17), 121(18), 104(24), 91\left(49,\left[\mathrm{C}_{7} \mathrm{H}_{7}\right]^{+}\right), 77$ $\left(29,\left[\mathrm{C}_{6} \mathrm{H}_{5}\right]^{+}\right), 76\left(25,\left[\mathrm{C}_{6} \mathrm{H}_{4}\right]^{+}\right), 69\left(17,\left[\mathrm{CF}_{3}\right]^{+}\right), 59\left(33,\left[\mathrm{C}_{3} \mathrm{H}_{7} \mathrm{O}\right]^{+}\right)$.

MS (CI, methane): $m / z=582\left(2,[\mathrm{M}+\mathrm{Et}]^{+}\right), 554\left(22,[\mathrm{M}+\mathrm{H}]^{+}\right)$.

HRMS (ESI): $m / z[\mathrm{M}+\mathrm{Na}]^{+}$calcd for $\mathrm{C}_{29} \mathrm{H}_{22} \mathrm{~F}_{3} \mathrm{NO}_{7} \mathrm{Na}^{+}: 576.1241$; found: 576.1241 .

(2S,3S,4S,5R,6R)-3-Benzoyl-2-hydroxy-5-nitro-4,6-diphenyl-2(trifluoromethyl)spiro[cyclohexane-1,2'-indene]-1',3'-dione (5n)

Yield: $163 \mathrm{mg}(54 \%, \mathrm{dr}=1.3: 1)$; colorless solid; 0\% ee [SFC: $(R, R)$ Whelk-01]; $R_{f}=0.19$ ( $n$-pentane-Et ${ }_{2} \mathrm{O}, 2: 1$ ).

IR (ATR): 3334, 3038, 2648, 2323, 2204, 2075, 1961, 1886, 1706, 1554, $1451,1357,1243,1168,1026,912,764,693 \mathrm{~cm}^{-1}$.

${ }^{1} \mathrm{H}$ NMR $\left(600 \mathrm{MHz}, \mathrm{CDCl}_{3}\right): \delta=7.95\left(\mathrm{~d},{ }^{3} \mathrm{~J}=7.9 \mathrm{~Hz}, 1 \mathrm{H}, \mathrm{ArH}\right), 7.92-$ $7.64(\mathrm{~m}, 12 \mathrm{H}, \mathrm{ArH}), 7.59\left(\mathrm{t},{ }^{3} \mathrm{~J}=7.7 \mathrm{~Hz}, 3 \mathrm{H}, \mathrm{ArH}\right), 7.44\left(\mathrm{t},{ }^{3} \mathrm{~J}=7.7 \mathrm{~Hz}, 2\right.$ $\mathrm{H}, \mathrm{ArH}), 7.35-7.20(\mathrm{~m}, 6 \mathrm{H}, \mathrm{ArH}), 7.18-7.00(\mathrm{~m}, 10 \mathrm{H}, \mathrm{ArH}), 6.93(\mathrm{t}$, $3 J=7.2 \mathrm{~Hz}, 3 \mathrm{H}, \mathrm{ArH}), 6.87$ (br s, $1 \mathrm{H}, \mathrm{ArH}), 6.63-6.51\left(\mathrm{~m}, 2 \mathrm{H}, \mathrm{CHNO}_{2}\right.$, $\mathrm{OH}), 6.34^{\mathrm{a}}(\mathrm{s}, 1 \mathrm{H}, \mathrm{OH}), 6.07^{\mathrm{a}}\left(\mathrm{dd},{ }^{3} \mathrm{~J}=11.4 \mathrm{~Hz},{ }^{3} \mathrm{~J}=7.9 \mathrm{~Hz}, 1 \mathrm{H}, \mathrm{CHNO}_{2}\right)$, $5.50^{\mathrm{a}}\left(\mathrm{d},{ }^{3} \mathrm{~J}=11.9 \mathrm{~Hz}, 1 \mathrm{H}, \mathrm{CHC}=0\right), 5.34\left(\mathrm{~d},{ }^{3} \mathrm{~J}=7.4 \mathrm{~Hz}, 1 \mathrm{H}, \mathrm{CHC}=0\right)$, $5.13\left(\mathrm{~d},{ }^{3} \mathrm{~J}=12.9 \mathrm{~Hz}, 1 \mathrm{H}, \mathrm{PhCH}\right), 4.97^{\mathrm{a}}\left(\mathrm{dd},{ }^{3} \mathrm{~J}=11.9 \mathrm{~Hz},{ }^{3} \mathrm{~J}=7.9 \mathrm{~Hz}, 1 \mathrm{H}\right.$, $\mathrm{PhCH}), 4.67^{\mathrm{a}}\left(\mathrm{d},{ }^{3} \mathrm{~J}=11.4 \mathrm{~Hz}, 1 \mathrm{H}, \mathrm{PhCH}\right), 4.47$ ('t', ${ }^{3} \mathrm{~J}=7.4 \mathrm{~Hz}, 1 \mathrm{H}$, $\mathrm{PhCH})$.

${ }^{13} \mathrm{C}$ NMR (101 MHz, $\mathrm{CDCl}_{3}$, C,F-decoupled): $\delta=202.6,200.4,200.2$, $198.2,196.3,194.5,143.2,142.9,141.2,137.6,136.7,136.4,136.2$, 135.8, 135.6, 135.2, 134.6, 133.9, 133.4, 133.1, 133.0, 129.6, 129.5, $129.1,129.0,128.9,128.8,128.7,128.7,128.6,128.5,123.7,123.6$, $123.5,123.1,91.6^{a}, 84.4,80.9^{a}, 79.8,62.7^{a}, 59.5,47.4,46.9^{a}, 44.4^{a}$, $43.2,42.5,41.5^{\mathrm{a}}$.

${ }^{19} \mathrm{~F} \operatorname{NMR}\left(376 \mathrm{MHz}, \mathrm{CDCl}_{3}\right): \delta=-71.0(\mathrm{~s}, 3 \mathrm{~F}),-71.9(\mathrm{~s}, 3 \mathrm{~F})$.
MS (EI, $70 \mathrm{eV}): m / z=431\left(1,\left[\mathrm{M}-\mathrm{NO}_{2}-\mathrm{PhCO}_{2} \mathrm{H}\right]^{+}\right), 347(1), 304(1)$, 249 (2), 233 (100), 216 (37), 176 (16), 147 (48), 105 (65), 91 (27, $\left.\left[\mathrm{C}_{7} \mathrm{H}_{7}\right]^{+}\right), 77\left(64,\left[\mathrm{C}_{6} \mathrm{H}_{5}\right]^{+}\right), 76\left(30,\left[\mathrm{C}_{6} \mathrm{H}_{4}\right]^{+}\right), 69\left(54,\left[\mathrm{CF}_{3}\right]^{+}\right), 65(11), 51$ (30).

MS (CI, methane): $m / z=600\left(11,[\mathrm{M}+\mathrm{H}]^{+}\right)$.

HRMS (ESI): $m / z[\mathrm{M}+\mathrm{Na}]^{+}$calcd for $\mathrm{C}_{30} \mathrm{H}_{24} \mathrm{~F}_{3} \mathrm{NO}_{7} \mathrm{Na}^{+}: 622.1448$; found: 622.1450 .

\section{Acknowledgment}

Financial support by the European Research Council (ERC grant 320493 'DOMINOCAT') is gratefully acknowledged. M.B. thanks Dr. C. Räuber for NMR measurements with a chiral shift reagent.

\section{Supporting Information}

Supporting information for this article is available online at http://dx.doi.org/10.1055/s-0035-1560072.

\section{References}

(1) For selected reviews and examples, see: (a) Enders, D.; Grondal, C.; Hüttl, M. R. M. Angew. Chem. Int. Ed. 2007, 46, 1570. (b) Walji, A. M.; MacMillan, D. W. C. Synlett 2007, 1477. (c) Yu, X.; Wang, W. Org. Biomol. Chem. 2008, 6, 2037. (d) Grondal, C.; Jeanty, M.; Enders, D. Nat. Chem. 2010, 2, 167. (e) Albrecht, Ł.; Jiang, H.; Jørgensen, K. A. Angew. Chem. Int. Ed. 2011, 50, 8492. (f) Alemán, J.; Parra, A.; Jiang, H.; Jørgensen, K. A. Chem. Eur. J. 2011, 17, 6890. (g) Moyano, A.; Rios, R. Chem. Rev. 2011, 111, 4703. (h) Loh, C. C. J.; Enders, D. Chem. Eur. J. 2012, 18, 10212. (i) Grossmann, A.; Enders, D. Angew. Chem. Int. Ed. 2012, 51, 314. (j) Lu, L.-Q.; Chen, J.-R.; Xiao, W.-J. Acc. Chem. Res. 2012, 45, 1278. (k) Pellissier, H. Adv. Synth. Catal. 2012, 354, 237. (l) Goudedranche, S.; Raimondi, W.; Bugaut, X.; Constantieux, T.; Bonne, D.; Rodriguez, J. Synthesis 2013, 45, 1909. (m) Wang, Y.; Xu, P.-F. In Catalytic Cascade Reactions; Xu, P.-F.; Wang, W., Eds.; Wiley-VCH: Weinheim, 2013, 123. (n) Volla, C. M. R.; Atodiresei, I.; Rueping, M. Chem. Rev. 2014, 114, 2390. (o) Chauhan, P.; Mahajan, S.; Kaya, U.; Hack, D.; Enders, D. Adv. Synth. Catal. 2015, 357, 253.

(2) (a) Kamigakinai, T.; Nakashima, M.; Tani, H. Japanese Patent JP 10101666 A2, 1998; Chem. Abstr. 1998, 129, 236773. (b) Kamigaichi, T.; Nakashima, M.; Tani, H. Japanese Patent JP 11158109 A2, 1999; Chem. Abstr. 1999, 131, 378444. (c) Wilson, K. E.; Tsou, N. N.; Guan, Z.; Ruby, C. L.; Pelaez, F.; Gorrochategui, J.; Vicente, F.; Onishi, H. R. Tetrahedron Lett. 2000, 41, 8705.

(3) Urata, H.; Kinoshita, A.; Misono, K.; Bumpus, F. M.; Husain, A. J. Biol. Chem. 1990, 265, 22348.

(4) Pizzirani, D.; Roberti, M.; Grimaudo, S.; Di Christina, A.; Pipitone, R. M.; Tolomeo, M.; Recanatini, M. J. Med. Chem. 2009, $52,6936$.

(5) For recent reviews on the importance of fluorinated compounds in pharmaceutical applications and their synthesis, see: (a) Wang, J.; Sánchez-Roselló, M.; Aceña, J. L.; del Pozo, C.; Sorochinsky, A. E.; Fustero, S.; Soloshonok, V. A.; Liu, H. Chem. Rev. 2014, 114, 2432. (b) Huang, Y.-Y.; Yang, X.; Chen, Z.; Verpoort, F.; Shibata, N. Chem. Eur. J. 2015, $21,1$.

(6) (a) Ramachary, D. B.; Chowdari, N. S.; Barbas, C. F. III Synlett 2003, 1910. (b) Ramachary, D. B.; Anebouselvy, K.; Chowdari, N. S.; Barbas, C. F. III J. Org. Chem. 2004, 69, 5838. (c) Ramachary, D. 
B.; Barbas, C. F. III Chem. Eur. J. 2004, 10, 5323. (d) Pizzirani, D.; Roberti, M.; Recanatini, M. Tetrahedron Lett. 2007, 48, 7120. (e) Ramachary, D. B.; Venkaiah, C.; Krishna, P. M. Chem. Commun. 2012, 48, 2252. (f) Luo, N.-H.; Sun, X.; Wei, W.-T.; Zhang, X.-J.; Yan, M. Tetrahedron: Asymmetry 2013, 24, 402. (g) Roy, S.; Amireddy, M.; Chen, K. Tetrahedron 2013, 69, 8751. (h) Kuan, H.-H.; Chien, C.-H.; Chen, K. Org. Lett. 2013, 15, 2880. (i) Anwar, S.; Li, S. M.; Chen, K. Org. Lett. 2014, 16, 2993. (j) Chen, R.; Xu, S.; Fan, X.; Li, H.; Tang, Y.; He, Z. Org. Biomol. Chem. 2015, 13, 398. (k) Han, B.; Huang, W.; Ren, W.; He, G.; Wang, J.; Peng, C. Adv. Synth. Catal. 2015, 357, 561.

(7) Dai, B.; Song, L.; Wang, P.; Yi, H.; Cao, W.; Jin, G.; Zhu, S.; Shao, M. Synlett 2009, 1842.

(8) Malerich, J. P.; Hagihara, K.; Rawal, V. H. J. Am. Chem. Soc. 2008, 130,14416

(9) For selected examples of squaramide-catalyzed Michael additions of dicarbonyl compounds to nitroalkenes, see: (a) Bae, H. Y.; Some, S.; Oh, J. S.; Lee, Y. S.; Song, C. E. Chem. Commun. 2011, 47, 9621. (b) Bae, H. Y.; Some, S.; Lee, J. H.; Kim, J.-Y.; Song, M. J.; Lee, S.; Zhang, Y. J.; Song, C. E. Adv. Synth. Catal. 2011, 353, 3196. (c) Wang, Y. F.; Chen, R.-X.; Wang, K.; Zhang, B.-B.; Lib, Z.-B.; Xu, D.-Q. Green Chem. 2012, 14, 893. (d) Chen, D.-F.; Wu, P.-Y.; Gong, L.-J. Org. Lett. 2013, 15, 3958. (e) Kótai, B.; Kardos, G.; Hamza, A.; Farkas, V.; Pápai, I.; Soós, T. Chem. Eur. J. 2014, 20, 5631.

(10) (a) Blümel, M.; Chauhan, P.; Hahn, R.; Raabe, G.; Enders, D. Org. Lett. 2014, 16, 6012. (b) Hahn, R.; Raabe, G.; Enders, D. Org. Lett. 2014, 16, 3636. (c) Chauhan, P.; Urbanietz, G.; Raabe, G.; Enders,
D. Chem. Commun. 2014, 50, 6853. (d) Urbanietz, G.; Atodiresei, I.; Enders, D. Synthesis 2014, 46, 1261. (e) Chauhan, P.; Mahajan, S.; Loh, C. C. J.; Raabe, G.; Enders, D. Org. Lett. 2014, 16, 2954. (f) Loh, C. C. J.; Chauhan, P.; Hack, D.; Lehmann, C.; Enders, D. Adv. Synth. Catal. 2014, 356, 3181. (g) Mahajan, S.; Chauhan, P.; Loh, C. C. J.; Uzungelis, S.; Raabe, G.; Enders, D. Synthesis 2015, 47, 1024. (h) Chauhan, P.; Mahajan, S.; Raabe, G.; Enders, D. Chem. Commun. 2015, 51, 2270.

(11) The enantiomeric excess of the Michael adduct 3a could not be determined by chiral stationary phase HPLC or SFC, but was estimated by NMR measurement with a chiral shift reagent to be approximately $60 \%$.

(12) A complete list of tested substrates can be found in the Supporting Information.

(13) (a) CCDC 1406112 (major diastereomer of 5b) and CCDC 1406111 (minor diastereomer of $\mathbf{5 b}$ ) contain the supplementary crystallographic data for this paper. The crystallographic data of both diastereomers of compound $\mathbf{5 b}$ are available free of charge at http://www.ccdc.cam.ac. (b) The unit cell of the major diastereomer of $\mathbf{5 b}$ includes an additional molecule of the same absolute configuration. For clarity only one molecule is depicted. Furthermore, the crystal structure of both diastereomers included one benzene molecule as solvent, which is also not depicted. Further details can be found in the Supporting Information. (c) The proposed mechanism and a model for the stereoselectivity can be found in the Supporting Information. 